\title{
Intensification studies of heterogeneous catalysts: probing and overcoming catalyst deactivation during liquid phase operation
}

\begin{abstract}
Ceri Hammond*a
In addition to high levels of catalytic activity and target product selectivity, promising heterogeneous catalysts must also possess sufficient levels stability for scale up, i.e. intensification, and industrialisation to be realised. However, this third often determining - keystone is often overlooked, particularly in the academic literature. This tutorial review therefore covers several elements related to the study of heterogeneous catalyst stability during liquid phase operation, an increasingly important area of heterogeneous catalysis. Particular emphasis is placed upon how stability data can be obtained, how the various forms of catalyst deactivation can be experimentally identified, and attention is drawn to emerging methods by which such events can be overcome or minimised. Drawing on some of our teams recent research, particular emphasis is placed on the stability studies of Lewis acidic silicates, state-of-the-art catalysts for a range of emerging liquid-solid catalytic processes. Factors related to heterogeneous nanoparticle catalysts are also covered. The purpose of this review is to draw attention to the issue of solid catalyst stability during liquid phase operation, and provide researchers with the information required to begin kinetic and spectroscopic studies of this determining catalytic event.
\end{abstract}

\section{Introduction}

Given the decreasing availability of traditional feedstock, issues associated with climate change, in addition to ever-increasing population growth, it is evident that the chemical industry needs to continue to increase its sustainability. ${ }^{1,2}$ Accordingly, energy consumption needs to be reduced; fossil fuel utilization should be minimised; production routes for chemicals and energy should be re-optimised, in light of decreasing feedstock availability; and the amount of waste co-produced should be decreased substantially. Given their ubiquity in all chemical processes, catalysts are able to influence each of these issues. Accordingly, the development of new catalysts, the improvement of existing catalysts, and the exploitation of catalysts on a greater scale, are tremendously important topics in modern research.

In heterogeneous catalysis, the catalyst is present as a solid phase, and reactants are present as gases or liquids. Such solid catalysts are widely employed at all levels of the chemical value chain, although they are especially employed in the bulk chemical industry. ${ }^{3,4}$ Traditionally, solid materials have been employed as heterogeneous catalysts for gas-solid (GS) reactions. This is largely due to the high volatility and thermal stability of crude oil, which continues to represent the major source of fuels and chemicals in the petrochemical industry. However, with increasing attention being focused on the sustainability of the chemical industry, coupled with the need

\footnotetext{
a. Cardiff Catalysis Institute, Cardiff University School of Chemistry, Main Building, Park Place, Cardiff, CF10 3AT, UK.

† Footnotes relating to the title and/or authors should appear here.
}

for raw material exchange i.e. the utilisation of renewable feedstock, there is an emergence of heterogeneous catalytic processes operating in a liquid-solid (LS), or gas-liquid-solid (GLS) regime. ${ }^{5-7}$ The development of such LS/GLS processes is co-driven by sustainability factors and necessity; by operating in the liquid phase, reaction temperatures and conditions can typically be milder than required for analogous GS catalytic processes; and emerging renewable feedstock are typically highly functionalised, oxygenated molecules derived from lignocellulosic biomass, and do not possess sufficient volatility nor thermal stability to be upgraded by conventional vapour/gas phase processes..$^{8,9}$ Moreover, such feedstock are typically produced via hydrolysis, and are therefore obtained as dilute aqueous/liquid streams. ${ }^{10-15}$

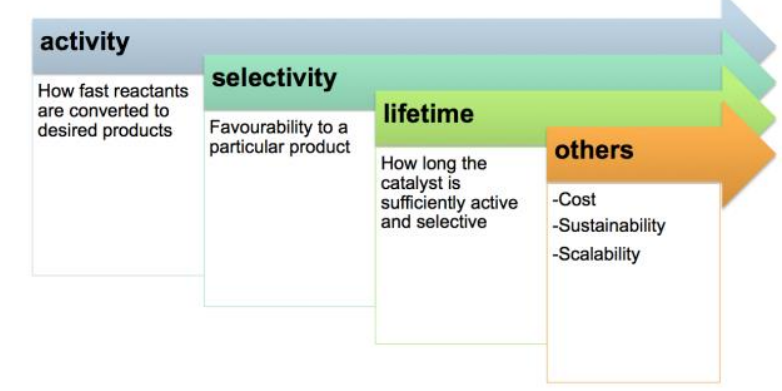

Figure 1. Properties a successful heterogeneous catalyst must possess.

The development of any new, successful catalytic processes is dependent upon sufficient performance in several key areas. Indeed, several particular performance indicators dictate the overall performance of a heterogeneous catalyst for a given chemical transformation, as outlined in Figure 1. 
A major performance indicator for a heterogeneous catalyst is evidently catalytic activity, that is to say the amount of product produced per unit time and per unit of catalyst. Typically, the catalytic activity of various materials is evaluated on the basis of the amount of substrate converted in a given unit time under a defined set of reaction conditions (Equation 1). Although such an evaluation allows one to quickly discriminate between active and less-active catalyst candidates, it should be noted that such a comparison is by definition arbitrary, and depends on the concentration of both the substrate and the catalyst. Indeed, by operating with highly diluted feeds and high masses of catalyst, extremely high values of conversion can be obtained even without a catalyst being particularly active or attractive from a commercial perspective. In addition to not providing a robust assessment of the potential of a catalytic material, such an approach can also lead to transport limitations (see 2.1). For these reasons, catalyst activity is more importantly measured by the metrics of spacetime-yield (STY, Equation 2) and catalyst productivity (Equation $3)$, which represent the overall productivity of the reactor and the catalyst, respectively. ${ }^{1}$ Occasionally, STY and catalyst productivity can be combined into one measurable value for the overall system productivity $(\Phi$, Equation 4$)$.

Important molecular information of the performance of particular heterogeneous catalysts can also be obtained by measuring the intrinsic performance of a catalytic material. Such a measurable, best classified in terms of turnover frequency (TOF), relates to the performance of the material based on the number of active sites per gram of material (Equation 5). Typically, the total metal loading is used to determine the TOF, particularly as determining the 'true' number of active sites per catalyst charge is a formidable challenge. Comparable information can also be obtained through detailed rate analysis (not written for brevity). However, it is important to add that intrinsic activity i.e. TOF, and any measurements of kinetic rate, should only be determined at the initial stages of the kinetic profile, where product-induced deactivation does not contribute to the observed kinetics.

$$
\text { Conversion }=X_{(t)}=\frac{\left([R]_{0}-[R]_{t}\right)}{[R]_{0}} \times 100(\%)
$$

(1)

$$
\begin{aligned}
& \mathrm{STY}=\operatorname{gram}_{\text {(product) }} \mathrm{cm}^{-3} \text { (reactor volume) } \\
& \text { Catalyst productivity }=\operatorname{gram}_{\text {(product) }} \mathrm{kg}^{-1} \text { (catalyst) } \\
& \Phi=\operatorname{gram}_{\text {(product) }} \mathrm{kg}^{-1} \text { (catalyst) } \mathrm{cm}^{-3} \text { (reactor volume) } \text { hour }^{-1} \\
& \text { TOF }=\text { moles }_{\text {(converted) }} \text { moles }^{-1} \text { (active site) } \text { hour }^{-1}
\end{aligned}
$$

Although catalyst activity is widely thought to be the most important performance indicator, it is rarely the defining property of an industrialised heterogeneous catalyst. Indeed, since downstream separation often represents a bigger financial and energetic cost than that of the main catalytic reaction itself, obtaining maximum selectivity to the target product (Equation 6), even at the expense of lower substrate conversion, is often more critical. ${ }^{16}$ In fact, several industrial catalytic processes operate at sub-maximal values of conversion in order to maintain sufficient selectivity, with increased conversions being obtained through reactant recycle loops. Obtaining high target product selectivity is even more critical when the feedstock costs are high, as is typically the case for emerging, non-crude oil-based substrates. ${ }^{16,17}$ It should be stressed, however, that product selectivity can vary substantially along the reaction coordinate. Consequently, selectivity should always be measured as a function of the reactant conversion. This is particularly important when consecutive or intermediate reactions may be present. Thus, comparing selectivity at different levels of conversion should always be avoided as it can lead to improper conclusions being made, and the selectivity obtained over different catalysts should always be compared at the same level of conversion.

$$
\begin{aligned}
\text { Selectivity }=S_{(A)}= & \frac{[P]_{(A)}}{\Sigma[P]_{(\text {total }}} \times 100=\frac{[P]_{(A)}}{\left([R]_{0}-[R]_{t}\right)} \times \\
& 100(\%) \text { (6) }
\end{aligned}
$$

In addition to possessing high levels of catalytic activity and excellent levels of target product selectivity, promising heterogeneous catalysts also need to demonstrate excellent levels of stability. In fact, the economic potential and sustainability of any possible catalytic process intimately depends on the ability of the catalyst to be reused (in the case of a batch system) or continuously used (in the case of a flow system) without loss in activity or overall performance over a substantial period of time. Although critical to the commercial prospects of a heterogeneous catalytic system, this performance indicator is often overlooked, especially in the academic literature. Particularly with the establishment of new liquid phase catalytic processes operating under nonconventional conditions (Vide Supra), ${ }^{18,19}$ the stability of heterogeneous catalysts for emerging types of chemical processes is far from understood, and consequently requires a great deal of focused study. ${ }^{20}$ Indeed, the presence of a caustic/acidic solvent, and the generation of hydrothermal conditions, can easily lead to destruction a heterogeneous catalytic material by mechanisms not prevalent during GS operation. ${ }^{21}$

The stability of a heterogeneous catalyst is typically evaluated in terms of the total number of turnovers obtained by a particular quantity of catalyst (expressed in terms of TON, Equation 7), or in terms of total catalyst productivity (Equation 8). Neither factor contains a "time" term. Accordingly, even poorly active catalysts can be of industrial relevance provided that they are sufficiently stable that over their period of operation a suitable number of turnovers can be obtained, although higher activity is of course always desirable.

TON $=$ moles $_{(\text {converted) }}$ moles $^{-1}{ }_{\text {(active site) }}$

Total catalyst productivity $=\operatorname{gram}_{\text {(product) }} \mathrm{kg}^{-1}$ (catalyst) 
In addition to activity, selectivity and stability - which represent the key performance targets for a potential heterogeneous catalyst - one must also consider the overall sustainability of the catalytic process in terms of its economics and scalability. One such consideration, which is the cost of the heterogeneous catalyst, is often discussed but is rarely the major bottleneck. Indeed, provided a heterogeneous catalyst is sufficiently stable, and that its active components can be recycled after its period of operation, then the cost of the catalyst elements can be considered a capital investment, and processing costs alone need be considered. That is not to say, however, that expensive elements can necessarily be used. Care must be taken to match the availability of the catalytic element to the scales required on a process level, and in the case of liquid phase heterogeneous catalysis, even small amounts of leaching (see 3.5) can result in a loss of expensive catalytic elements, and a significant increase in production costs. ${ }^{22}$ Once more, the stability of a heterogeneous catalyst in terms of its ability to perform for a sufficient period of time becomes the critical factor.

\section{Determining catalyst stability}

In order to rigorously assess the stability of any particular heterogeneous catalyst, a large number of accurate kinetic experiments need to be performed. In this section, we discuss the various experiments that should be undertaken during these studies, and outline the main considerations that should be addressed when exploring the stability of heterogeneous catalysts during liquid phase operation. Drawing on our recent studies of Lewis acidic silicates, the methods measurements described herein can be applied to almost any heterogeneous catalytic material performing liquid phase catalytic chemistry.

2.1 Determining catalyst stability in batch reactors. Most studies focusing on the stability of heterogeneous catalysts for LS operation typically perform recyclability tests. In such experiments, the catalyst is filtered out of the reaction solution after one batch reaction, and is then re-evaluated in a second catalytic reaction containing a fresh amount of reaction solution. Comparison of the maximal conversion obtained in each cycle is then used to assess the re-usability of the catalyst. According to this method, preliminary information on the stability of a promising heterogeneous catalyst can be obtained. For example, several research groups, including ours, have demonstrated that the Lewis acidic silicate, zeolite Sn- $\beta$, exhibits high levels of stability during batch operation. Examples include its stability during the conversion of sucrose to methyl lactate, the isomerisation of various sugars, the MeerweinPondorf-Verley transfer hydrogenation of carbonyl compounds, and the Baeyer-Villiger oxidation of various ketones. ${ }^{23-25}$ In each case, little or no loss in maximal conversion was observed in each of these reports even after $<6$ successive catalytic experiments (Figure 2 ).

Although useful as a first piece of information, several issues limit the suitability of these studies for probing catalyst stability.
Firstly, comparing catalytic activity at high (often maximal) conversion does not allow the intrinsic kinetic behaviour of the catalyst during each cycle to be compared. Thus, changes in kinetic behaviour can be missed. An illustrative time online profile of three successive catalytic cycles is provided in Figure 3. In this case, comparing the maximal conversion obtained after $4 \mathrm{~h}$ during each cycle would give the incorrect impression that the activity of the catalyst is stable, when the initial rates (at $0.5 \mathrm{~h}$ ) are very different. Secondly, given that batch experiments are typically performed with low substrate/metal ratios, recyclability testing often only results in a low number of total turnovers being observed. For example, during the BaeyerVilliger oxidation (BVO) of cyclohexanone with $\mathrm{H}_{2} \mathrm{O}_{2}$, three successive catalytic experiments over $\mathrm{Sn}-\beta$ zeolite resulted in less than 350 turnovers, far below the typical number required for intensification-based studies. ${ }^{26}$ Another major limitation of recyclability studies is that periodic regeneration of the catalyst by washing and/or re-calcination, as is typically performed, does not mimic true operation. Indeed, performing such reactivation protocols between successive catalytic cycles can easily mask deactivation effects that occur during each individual batch run.

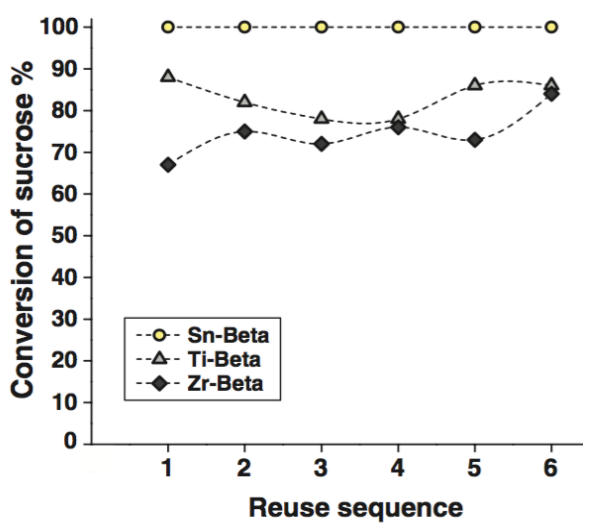

Figure 2. Recyclability studies of Sn-, Ti- and Zr- $\beta$ during the catalytic formation of methy lactate from sucrose. Figure reproduced with permission from reference 23.

This is not to say that recyclability studies are not informative, however. In fact, provided certain control experiments are performed, they can provide a rapid, preliminary assessment of catalyst stability. For recyclability studies to be fully relevant, therefore, several factors must be remembered. Firstly, the activity of a catalyst, particularly in terms of recyclability, should always be evaluated through full kinetic analysis, i.e. time online analysis should always be performed (Figure 3 ). This allows one to compare both maximal activity in terms of conversion, and also intrinsic activity in terms of TOF. This ensures potential changes to a particular active site ensemble can be readily identified even without extended time on stream catalytic testing (see 2.2). 


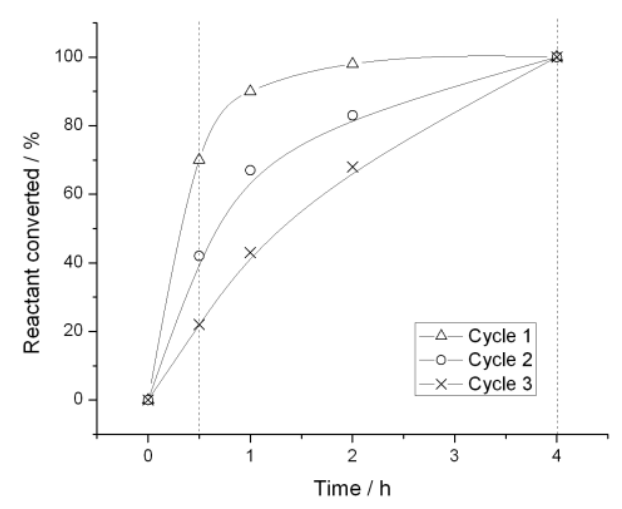

Figure 3. Illustration of the poor conclusions that can be obtained via recyclability studies performed at maximal levels of conversion.

Secondly, it must be remembered that intrinsic kinetic data can only be obtained from the kinetic regime of the system, i.e. control experiments to ensure the absence of transport limitations should always be performed. The simplest way of determining this is to perform a series of catalytic experiments in the presence of different masses of catalysts; in the kinetic regime, increasing the mass of catalyst should lead to a corresponding increase in the rate of reaction. If no increase is observed, then the amount of catalyst should be lowered until a linear relationship between catalyst concentration and rate is observed. Only under these conditions can true kinetic information be observed, and true comparison of catalytic performance as a function of reuse cycle be made.

Thirdly, even small amounts of leached metal species can easily show high levels of activity for a particular catalytic transformation. ${ }^{27}$ Indeed, even trace i.e. ppb, levels of leached metal ions from a heterogeneous catalyst can be responsible for high levels of catalytic performance. ${ }^{28,29}$ As such, it is important to rule out that successful repeated performance is not solely due to a small part of the active site of the heterogeneous catalyst leaching into solution and catalysing the reaction during successive cycles.

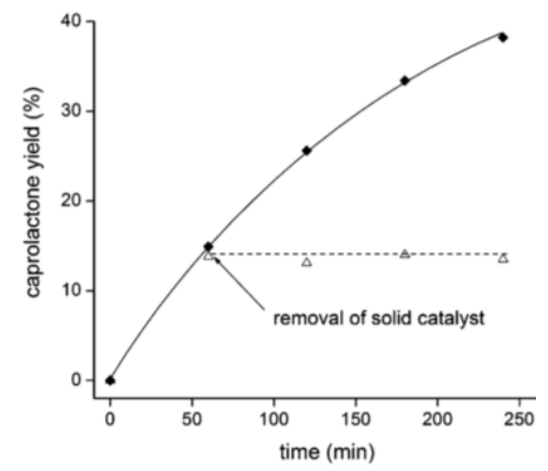

Figure 4. Kinetic example of hot filtration experiment that shows leaching is not responsible for the kinetic performance of a material. Figure reproduced with permission from reference 25 .

Accordingly, recyclability studies must always be accompanied by hot filtration experiments (Figure 4). ${ }^{25}$ In this two-step experiment, a typical catalytic experiment with time online analysis is first performed. Subsequently, a second amount of fresh catalyst is screened for catalytic activity. In this second test, however, the catalyst is filtered out of the reaction solution after a given period of time, and the remaining supernatant solution is allowed to continue reacting in the absence of the solid catalyst under otherwise-identical conditions. If the solid catalyst alone is responsible for activity, then the reaction should be terminated by removal of the solid catalysts, as was observed during our studies of $\mathrm{Sn}-\beta$ catalysed BVO (Figure 4). In contrast, should leached species be responsible for catalytic activity then the kinetic profile will continue to show an increase in conversion even after removal of the catalyst. Comparison of the extended time online profile of both reactions thus allows one to discriminate the extent that leached species play a role in catalysis. It should be noted, however, that a negative hot filtration experiment alone does not mean leaching has not occurred, it simply means that any potential leached species are not active homogeneous catalysts for the reaction.

Finally, it should be stressed that recyclability studies should be performed both with, and without, intermediate reactivation procedures between successive cycles. This is to understand how potential deactivation processes may occur during the recyclability series over a limited number of turnovers. Such reactivation procedures include washing the sample with a solvent, treating the sample with an appropriate wash solution, and heat treatments.

2.2 Determining stability in continuous reactors. A more rigorous method of probing catalyst stability is to perform the catalytic experiments in a continuous manner. Continuous Plug Flow Reactors (PFR, Figure 5) provide several major advantages compared to batch reactors, including i) improved process- and safety-control, ii) higher levels of mass- and heat-transfer, iii) faster rates of reaction, iv) minimised reactor volumes, iv) higher levels of scalability and v) improved space-time-yields. More critically, continuous PFRs also permit steady-state operation and rigorous assessment of catalyst stability..$^{30}$ In steady state operation, the concentration at any point along the reactor axis is invariant with time. ${ }^{31}$ As such, a continuous flow of reactant solution is fed over a given mass of catalytic material, and performance is monitored as a function of time on stream..$^{32}$ In this case, the contact time (the amount of time the reactant solution is in contact with the catalyst) and its reciprocal, Liquid Hourly Space Velocity (LHSV, the fraction of feed exposed to a given quantity of catalyst per unit time) are fixed based on the volume of the catalyst bed and the flow rate of the reactant solution (Equations 9-10). Both contact time and LHSV are important measures for comparing the performance of catalytic materials, i.e. obtaining a comparable conversion at a shorter contact time or larger LHSV confirms that a catalyst is more active. Hence, the reactant conversion obtained over a catalyst in a continuous reactor should always be reported as a function of contact time or LHSV.

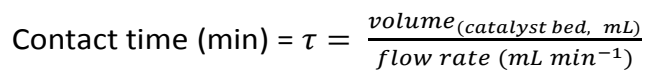


LHSV $=$ flow rate $_{(\text {feed solution })}$ volume $^{-1}$ (catalyst) hour $^{-1}$

By performing catalytic measurements under steady state conditions, catalyst deactivation can be probed more accurately and over a larger range of TON than during recyclability measurements. Moreover, potential deactivation processes are not masked by periodic regeneration, and leached metal species - which can lead to homogeneous background activity in batch vessels, see 2.1 - are washed out of the catalyst bed, and do not contribute to the on-going catalytic reaction. Moreover, the mode of operation is more comparable to that observed in an industrial catalytic reactor, and the activity and stability data can also be scaled more appropriately. When combined with spectroscopic methodologies that allow the physico-chemico properties of the material pre- and postreaction to be characterised (see section 3 ), it is a particularly powerful and accurate method of rigorously assessing catalyst stability.

Although full discussion over the type and classification of steady state catalytic reactors is beyond the scope of this review, ${ }^{33}$ a schematic of a PFR operating in LS mode, suitable for the study of heterogeneous catalysts during liquid phase catalysis is provided (Figure 5). Additionally, some experimental guidelines for obtaining kinetic data from such reactors are provided.

For true plug flow conditions to be observed in a LS reactor, the reactor diameter should be at least 10 times larger than the catalyst particle diameter. Additionally, the catalyst bed should be at least several hundred particle diameters long. In the case of Lewis acidic silicates catalysing liquid phase processes, reactors with an internal diameter (I.D.) between 1.5-6.3 mm, and several 10 's or 100's of $\mathrm{mm}$ of length, are typically employed, depending on the minimum charge of catalyst required to avoid interphase limitations (Vide Infra). Given their small particle sizes (typically $\leq 1 \mu \mathrm{m}$ ), the flow of the reactant feed through the packed catalyst bed comprising a Lewis acidic silicate may result in excessive pressure drops. To minimise this, and potential back mixing, one may choose to shape the catalyst particles, $^{34}$ or dilute the catalyst powder with an inert substance. ${ }^{35}$ In our studies, quartz or SiC are employed. ${ }^{36}$ Pressure in the reactor can be maintained by means of a backpressure regulator, and an HPLC or piston pump allows reactant delivery. Heating of the reactor column can be achieved through use of an appropriate furnace, or alternatively through immersion in a thermostatted oil bath.

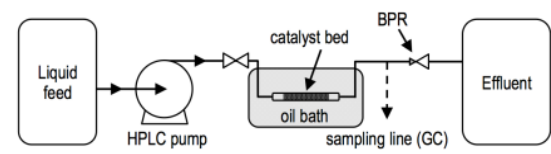

Figure 5. Schematic of a LS PFR for the continuous evaluation of catalytic activity.

Prior to extended time on stream measurements, it is important to perform several control experiments to rigorously assess the kinetic relevance of the LS PFR, i.e. it is important to verify that true kinetic data is being obtained.
One of the major conditions for observing good kinetic behaviour is to ensure the absence of various interphase limitations, such as external and internal mass transfer. To rule out potential external mass transfer limitations, the effect of linear velocity on catalytic activity needs to be determined. This involves preparing a range of catalyst columns of various lengths (diameter should be kept constant), and monitoring the catalytic activity observed at a fixed contact time, i.e. the flow rate should also be adjusted so that the overall contact time is kept constant. According to film theory, the liquid film around the catalyst particle becomes thinner as flow rate is increased, thus enhancing external mass transfer. As such, if external mass transfer is rate limiting then an increase in conversion will occur with increased flow rates. ${ }^{22}$ On the other hand, if conversion is independent of flow rate at a fixed contact time then the system is operating in the kinetic regime. As we recently reported (Figure 6A), maintaining a flow rate of at least $0.1 \mathrm{~mL} \mathrm{~min}^{-1}$ was essential for good kinetic behaviour to be observed during the Meerwein-Pondorf-Verley transfer hydrogenation of cyclohexanone, catalysed by $\mathrm{Sn}-\beta .{ }^{36}$ Depending on the results of this test, a minimum flow rate and mass charge of catalyst can be calculated.
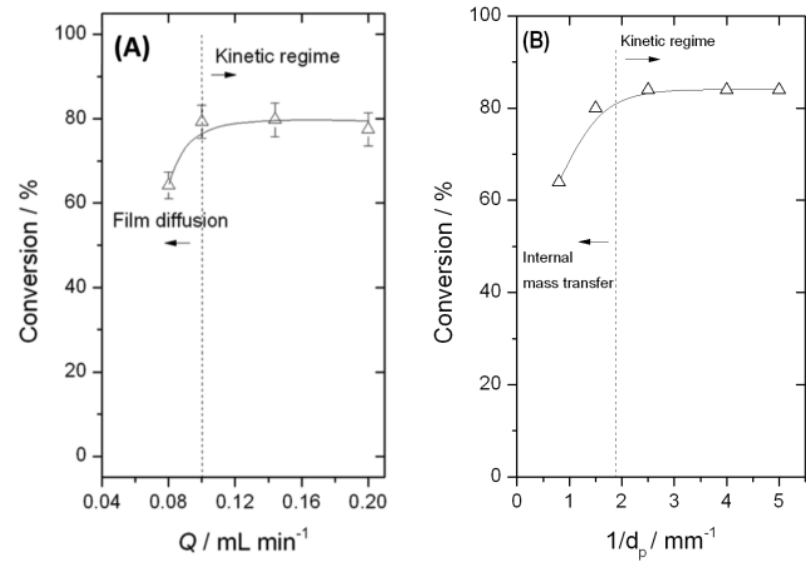

Figure 6. (Left) Effect of linear velocity, in terms of $\mathrm{mL} \mathrm{min}^{-1}$, on the conversion of cyclohexanone observed over Sn- $\beta$ during MPV transfer hydrogenation. (Right) Illustration of how catalyst particle size influences catalytic performance. Figure 6 (left) reproduced with permission from reference 36 .

In addition to external mass transfer, one should also consider the impact of internal mass transfer. Although the small crystallite size of Lewis acidic silicates typically results in the absence of internal mass transfer limitations, they can become apparent for bigger catalyst particles where the diffusion path length is large. The simplest way of verifying the absence of internal mass transfer in a LS PFR is to measure the conversion observed for a given amount of catalytic material, under a given set of conditions, as a function of particle size. In the absence of internal mass transfer limitations, the observed conversion should be independent of particle size. However, where internal mass transfer is observed, conversion will decrease. A plot of conversion versus the inverse of particle size $\left(1 / d_{p}\right)$ under otherwise identical conditions allows one to readily detect if two regimes are present (Figure 6, Right). This approach was employed by Aellig and co-workers to 
demonstrate the presence of internal mass transfer limitations during the liquid phase dehydration of fructose to 5hydroxymethylfurfural, when the reaction was catalysed by larger particles of Amberlyst-15. ${ }^{37}$

Once the reaction conditions have been optimised to ensure the absence of interphase limitations, a final method of confirming kinetic relevance of a PFR is to obtain an Arrhenius plot by measuring catalytic activity over a range of contact times and temperatures in the PFR. If suitable kinetic behaviour is observed, the activation energy obtained in the PFR should match that obtained at the same temperature range in a batch reactor. ${ }^{36}$

Provided good kinetic behaviour in the PFR has been identified, time on stream analysis and true elucidation of catalyst stability can be performed. Some key considerations that must be adhered to, however, include the following;

1. The contact time should always be adjusted so that some of the rate limiting reactant is always present, i.e. conversion should always be below the maximum equilibrium level. This is to ensure that an 'excess catalyst' regime is avoided, and true deactivation can be monitored. In the presence of excess catalyst, a significant amount of deactivation could occur before the conversion of a system decreases below $100 \%$. Such an approach is, however, regularly employed in industrial reactors in order to maximise the time between catalyst regenerations. ${ }^{38}$

2. When two different catalysts are being compared for long-term stability, the contact time in both tests should be adjusted so that the initial activity - in terms of conversion - is close to equal for each catalyst. This means both catalysts are tested at the same stage of the reaction profile, i.e. both catalysts are exposed to similar ratios of reactants and products. This is to ensure that particular product-induced deactivation events do not overly affect the more active catalyst under otherwise identical reaction conditions.

3. When comparing the stability of various catalysts, it is sometimes useful to compare activity not in terms of time on stream, but in terms of substrate turnovers $(\rho$, Equation 11). This is especially useful when comparing two catalysts of disparate activity, which convert a different amount of product per unit time, which is a more important measure of performance.

$p=\frac{n(\text { reactant }) \text { per } \min }{n(\text { moles active centre })} \cdot \Sigma_{t}$

\section{Identifying and overcoming particular deactivation phenomena}

Once suitable time on stream testing can proceed, one can start to assess the stability of particular heterogeneous catalysts, and critically gain an insight into why deactivation inevitably occurs. catalysts. Such processes can be grouped into two major categories: reversible and irreversible. ${ }^{21}$ In the first instance, the cause of deactivation is not permanent, and downstream processing of the catalyst by particular methods can restore full, or substantial, levels of activity. Although reversible, the length of time a catalyst may operate without requiring regeneration remains a critical parameter, as regeneration leads to unwanted reactor downtime, a corresponding decrease in space-timeyield, and can significantly impact the specific design of the catalytic reactor. ${ }^{38}$ On the other hand, irreversible deactivation is by definition permanent, and results in loss or destruction of the catalytic material. Some common processes known to lead to catalyst deactivation in conventional i.e. GS operation, include; poisoning by particular reactant/product/by-product species; fouling through the accumulation of reaction residue; active site reorganisation, either through sintering or gradual/catastrophic restructuring under the reaction conditions; thermal dissolution of the catalyst; and mechanical and thermal attrition of the catalyst powder under the harsh processing conditions. ${ }^{21}$

In addition to these factors - which can also occur during LS catalytic transformations - two further issues can impact the stability of a heterogeneous catalysts in a liquid medium; Leaching i.e. extraction, of the active phase from the solid catalyst into the reaction medium, and hydrothermal dissolution of the catalyst through processing in the reaction solvent. ${ }^{39}$ In addition to minimising the scalability of a process, leaching in particular can also lead to severe environmental implications in the form of toxic metal contamination, leading to unwanted and economically undesirable downstream processing requirements.

Needless to say, developing materials that are resistant to permanent deactivation is essential for the sustainability and scalability of a catalytic process. Identifying the cause(s) of deactivation, and devising strategies to minimise (or avoid) its impact, are therefore critical areas of catalysis research.

In the following sections, we describe the nature of catalyst deactivation in each of these categories, describe some of the experimental methods by which their presence can be identified, and highlight some of the emerging methodologies by which these deactivation events can be avoided or minimised. As illustrated in Figure 7, we will discuss the following causes of deactivation; (1) fouling, (2) poisoning, (3) active site reorganisation, (4) leaching, and (5) hydrothermal dissolution. It is important to add, however, that catalyst deactivation may not related to a single event or mechanism, and in many cases several particular deactivation process contribute to a global loss in activity.

3.1 Overview of catalyst deactivation processes. Several processes can lead to the deactivation of heterogeneous

\begin{tabular}{|l|lllll|}
\hline $\begin{array}{c}\text { Cause of } \\
\text { deactivation }\end{array}$ & \multicolumn{1}{|c|}{ Fouling } & \multicolumn{1}{c|}{$\begin{array}{c}\text { Poisoning } \\
\text { Deaching }\end{array}$} & $\begin{array}{l}\text { Active site re- } \\
\text { organization }\end{array}$ & $\begin{array}{c}\text { (Hydro)thermal } \\
\text { dissolution }\end{array}$ \\
\hline Description & & & & &
\end{tabular}


Figure 7. Overview to the type of deactivation events experienced by heterogeneous catalysts during liquid phase operation, along with methods of detection.

3.2 Fouling. Fouling is a generic term describing the deposition of carbonaceous residue onto the surface of, or within the pores of, a heterogeneous catalyst. The deposition of which subsequently blocks access of the reactants to the catalytic active sites. Observed in both gas phase and liquid phase processes, fouling is a challenge faced by all heterogeneous catalysts. Typically, fouling is related to the deposition of high molecular weight, polymeric carbonaceous species. Generally categorised as "coke", the precise nature of the carbonaceous residue responsible for fouling varies widely, and intimately depends on the nature of the reaction undergoing study, the chosen reaction conditions, the identity of the catalytic material, along with the nature of the feed solution. ${ }^{40}$

Although the generation of such species can be catalytic i.e. in addition to catalysing the main catalytic reaction, active sites on the heterogeneous catalyst also catalyse further polymerisation of a particular reaction (by-)product, the formation of such polymeric species does not necessarily relate to the catalytic chemistry undergoing study. The presence of poorly soluble components of the feed solution, e.g. the presence of humins in saccharide solutions during biomass upgrading, or the autogenic decomposition of the feedstock, e.g. the caremelisation of aldoses and ketoses, can also lead to fouling. As such, fouling can arise from (1) heavy components already present in the feed; (2) reactant components poorly soluble in the chosen solvent or (3) particularly unstable (by)products produced in the presence of the catalyst. ${ }^{41,42}$

The formation of such carbonaceous residue can lead to deactivation of a catalytic process through several types of mechanisms, including (1) chemical deactivation of the active site through poisoning, (2) physical plugging of the reactor channels, or more commonly (3) blocking the access of reactants to the active sites, either by encapsulation of the active site (particularly problematic for supported metal nanoparticles) or pore blocking. For Lewis acidic silicates and analogous microporous materials, the distribution of active sites throughout the microporous architecture makes them extremely susceptible to increased rates of deactivation through pore blocking. ${ }^{21}$ Kinetically, fouling can result in either a gradual or catastrophic decrease in catalytic activity, depending on the mechanism of carbonaceous residue formation and the nature of the active sites of the catalyst. Accordingly, spectroscopic characterisation of the catalytic material prior to, and following, continuous operation represents the best way of identifying fouling.

Given that fouling relates to the formation of carbonaceous residue trapped within the bulk, or on the surface of, the heterogeneous catalyst, thermogravimetric methods e.g. TGA, and hyphenated analogues thereof (TGA-MS, TGA-IR), represent one of the simplest forms of identification. Depending on the nature of the particular carbonaceous residue, the precise desorption temperature can vary substantially. Higher molecular weight fragments, such as humins, are generally characterized by an exothermic desorption at relatively high $\left(>250{ }^{\circ} \mathrm{C}\right)$ temperatures. In contrast, smaller molecular weight fragments may be observed at milder temperatures. For example, Koehle et al. demonstrated that during the reductive valorization of furfural over $\mathrm{Sn}$-, $\mathrm{Zr}$ - and Hf-cotaining zeolite Beta, the major cause of fouling was the retention and subsequent polymerization of furfuryl alcohol. ${ }^{43}$ In this case, retention of furfuryl alcohol within the pores was identified through a characteristic desorption at $390 \mathrm{~K}$. However, higher molecular weight oligomers from furfuryl alcohol were also observed at of \pm 800 $\mathrm{K}$ (Figure 8). It is notable that $\mathrm{CHN}$ analysis, and characterization of the post-catalytic material by Raman spectroscopy, can also provide complementary, information as to the presence of carbonaceous residue. Although fouled species may be insoluble under the particular reaction conditions, they may prove to be soluble in different solvents. Accordingly, product extraction methodologies (discussed in detail in 3.3) may also be of use.

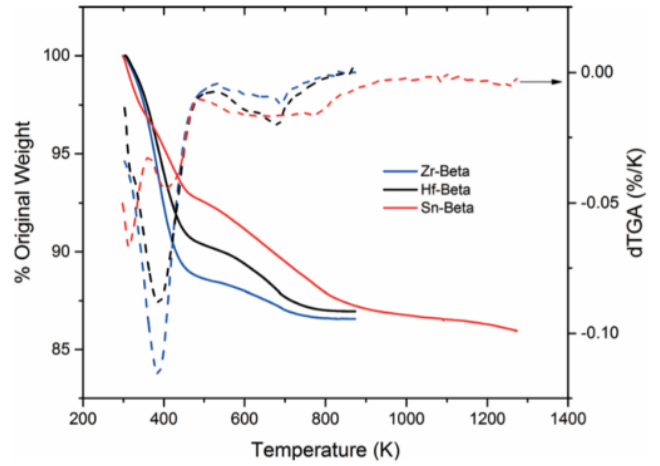

Figure 8. TGA measurements of $\mathrm{Zr}-, \mathrm{Hf}-$ and $\mathrm{Sn}-\beta$ following the liquid phase reductive valorisation of furfural. Reproduced with permission from reference 43.

For Lewis acidic silicates, porosimetric measurements of the used catalytic material also represent a valuable method of confirming fouling during extended operation. Although large decreases in specific surface area may not be observed, detailed measurement of the micropore volume prior to, and following, catalysis can lead to immediate identification of fouling. For example, our group identified that the micropore volume of $\mathrm{Sn}$ $\beta$ decreased from $0.23 \mathrm{~cm}^{3} \mathrm{~g}^{-1}$ to $0.11 \mathrm{~cm}^{3} \mathrm{~g}^{-1}$ after $550 \mathrm{~h}$ on stream during the MPV transfer hydrogenation of various carbonyl compounds such as cyclohexanone (Figures 9-10). ${ }^{36}$
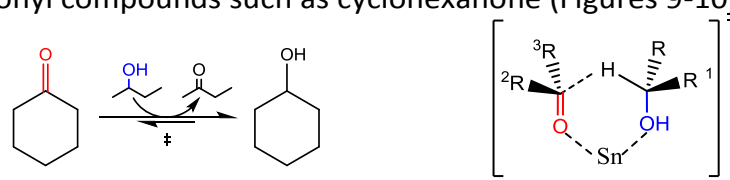
Figure 9. Catalytic transfer hydrogenation of various carbonyl compounds as catalysed by Sn- $\beta$. A six membered transition state involving coordination of the substrate and solvent (in this case, 2-butanol) mediates intermolecular hydride transfer.

Fouling is typically non-permanent in nature, and the carbonaceous residue formed can typically be removed during a subsequent heat-treatment. For example, we demonstrated that the catalytic activity of $\mathrm{Sn}-\beta$ could be fully restored after $550 \mathrm{~h}$ on stream by heat treatment in air at $550{ }^{\circ} \mathrm{C}$ (Figure 10). However, it should be emphasised that regeneration protocols lead to unwanted downtime and lead to a corresponding decrease in space-time-yield. ${ }^{38}$ Moreover, they may directly impact the design of the catalytic reactor, by requiring provision for high temperature heating that is evidently not required during liquid phase operation, or the ability to recycle large amounts of catalytic material. Additionally, certain catalysts such as metal nanoparticle-based catalysts, may undergo extensive sintering at the temperatures required for carbonaceous residue removal, and carbon-based materials are evidently unsuitable for high temperature thermal treatment under such conditions. ${ }^{44}$ Accordingly, methods of minimizing fouling during continuous operation, and the development of novel regeneration methods, such as washing and low temperature heat treatments (see section 4), are extremely important areas of research.

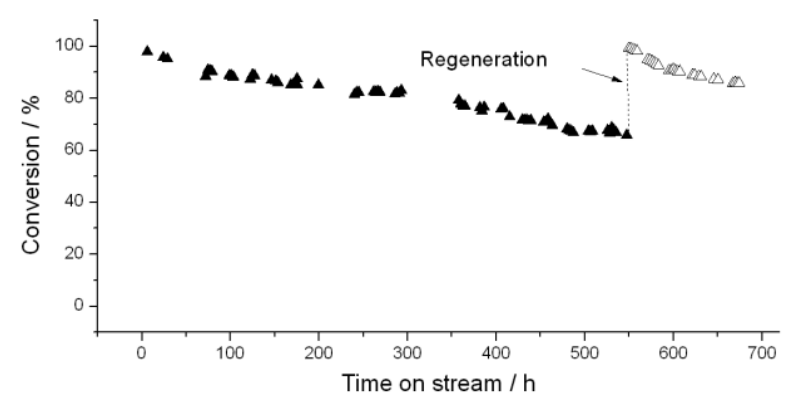

Figure 10. Stability of zeolite Sn- $\beta$ during the MPV transfer hydrogenation of cyclohexanone with 2-butanol as $\mathrm{H}$-donor. A regeneration protocol after $550 \mathrm{~h}$ is found to restore full levels of performance. Adapted with permission from reference 36 .

Pre-treatment of the reactant feed is one method of mitigating fouling. Removal of the heavy components, or use of a more suitable (co-)solvent to ensure their solubility, can readily result in minimized fouling. ${ }^{45}$ Moreover, where fouling is related to the polymerization of particular feed components, further dilution of the feed may also alleviate fouling by minimizing self-polymerization. However, excessive dilution of the feed may not be desired from an economic perspective, as it rapidly increases the cost of separation, and negatively impacts space-time-yield.
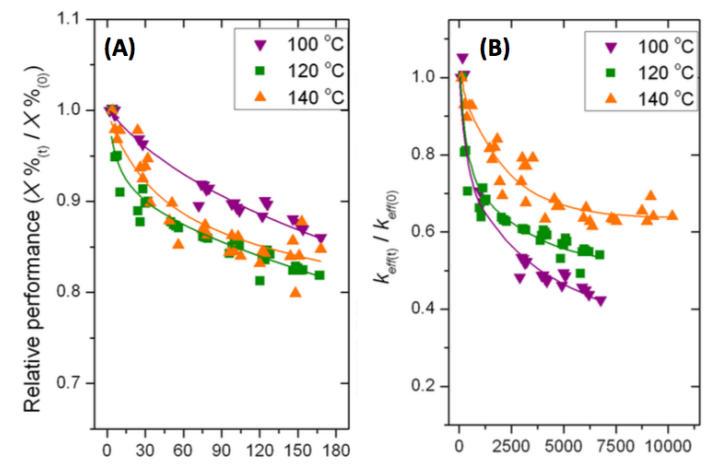

Figure 11. Catalytic stability of $\mathrm{Sn}-\beta$ as a function of (A) time on stream, and (B) number of substrate turnovers, during the MPV transfer hydrogenation of cyclohexanone over Sn- $\beta$ between 100 and $140^{\circ} \mathrm{C}$.

Modification to the reaction conditions can also lead to a significant decrease in fouling. For example, we recently demonstrated that increasing the reaction temperature from $100-140{ }^{\circ} \mathrm{C}$ aided desorption of the target reaction products during MPV transfer hydrogenation (the corresponding alcohols), thus decreasing their retention - and subsequent polymerization - within the structure of Lewis acidic zeolites. In addition to increasing product desorption and minimising fouling, the increase in temperature also led to a three-fold increase in productivity on a space-time-yield basis. Accordingly, deactivation as a function of turnover number (Figure 11B) was substantially minimized. ${ }^{36}$

In addition to tailoring the reaction conditions, it may also be possible to tailor the catalytic material itself to minimize fouling. For example, for microporous materials such as Lewis acidic zeolites, one possible mitigation strategy is to modify the overall porous architecture of the material in order to improve the rates of diffusion of particular reaction products prone to fouling. In recent years, several approaches have been followed, such as the synthesis of extra-large pore zeolites, the development of ordered mesoporous materials (e.g. MCM-41) and related composites, (3) the preparation of nano-sized zeolite particles, characterized by shorter diffusion path lengths, (4) the development of 2D materials zeolites and (5) the generation of hierarchical zeolites. ${ }^{46-52}$ Although several of these approaches have been followed for Lewis acidic zeolites, few studies have focused upon continuous operation with such materials.

We recently demonstrated conversion of a $\beta$ zeolite framework to a hierarchical structure through treatment in dilute $\mathrm{NaOH}\left(0.2 \mathrm{M}, 45{ }^{\circ} \mathrm{C}, 30\right.$ mins) readily improved continuous performance. ${ }^{53}$ Hierarchical frameworks possess both microporosity and mesoporosity, but retain the crystalline framework structure and the active site speciation. Indeed, caustic treatment of the $\beta$ zeolite lead to a five-fold increase in the mesoporous volume of the material $\left(V_{\text {meso }}>0.5 \mathrm{~cm}^{3} \mathrm{~g}^{-1}\right)$ without excessive destruction of the microporous structure, as evidenced by powder XRD measurements. Pores of approximately $8 \mathrm{~nm}$ were also observed by porosimetric measurements (Figure 12) and TEM. Thus, whilst intrinsic kinetic behaviour in terms of specific site activity, i.e. TOF, was largely unaffected, catalyst stability improved substantially. Over a period of $700 \mathrm{~h}$ on stream, the hierarchical Sn- $\beta$ was found to lose only $\pm 20 \%$ of its maximal performance. In 
contrast, microporous $\mathrm{Sn}-\beta$ was found to deactivate rapidly under the chosen conditions, losing $>60 \%$ of its activity in a period of less than $200 \mathrm{~h}$. In addition to minimising fouling, the hierarchical material was also able to catalyse the conversion of molecules too bulky to fit within the standard framework, thus improving the general applicability of the catalyst.

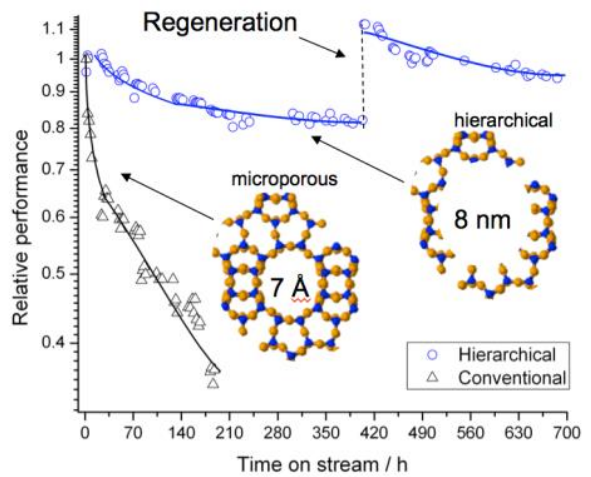

Figure 12. Relative performance of microporous (triangles) and hierarchical (circles) Sn$\beta$ during continuous MPV transfer hydrogenation $100^{\circ} \mathrm{C}$. Adapted with permission from reference 53 .

A major challenge in this area, however, is obtaining improved porosity without overly affecting the performance of the intrinsic active site in terms of TOF. For example, the intrinsic activity of Lewis acidic heteroatoms is reportedly lower when they are hosted in a more accessible, delaminated structures, as opposed to a conventional 3D frameworks. ${ }^{54,55}$ Furthermore, whilst caustic treatment of is a useful method of generating mesopores, it can reduce the quantity of active metal per gram of catalyst if performed after inclusion of the active component, hence compromising metal loading and consequently space-time-yield. As such, post-synthetic zeolite functionalisation strategies hold particular promise. In these circumstances, functionalisation of a pre-modified, hierarchical zeolite structure to include the active sites at the latest stage of synthesis is followed. Two key examples include alkalineassisted metallation, ${ }^{56}$ and the solid state incorporation of Lewis acidic heteroatoms into dealuminated zeolites. ${ }^{57}$ In both of these cases, introduction of the active component is achieved following structural modification of the zeolite, and active site performance (in terms of TOF) is not overly compromised. Moreover, positive enrichment of the zeolite with active sites on the external edges of the crystallites may be observed, leading to improved reactant/active site interactions, and no decrease in active site concentration is observed. A schematic of the solid state stannation of hierarchical $S n-\beta$ is provided (Figure 13).

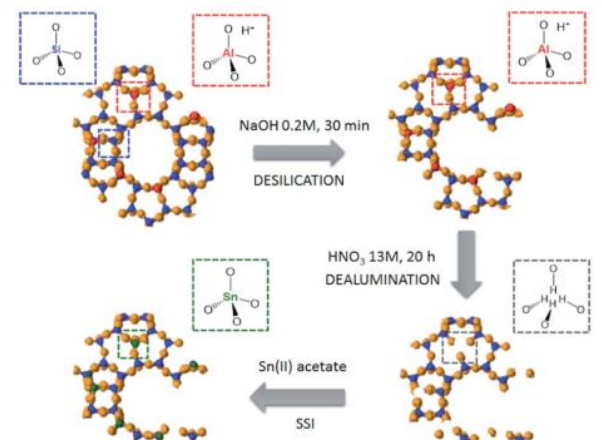

Figure 13. Post-synthetic procedure for the synthesis of hierarchical Sn- $\beta$, where active site incorporation is achieved at the final stage of synthesis. Adapted with permission from reference 53 .

3.3 Poisoning. Poisoning is the deactivation of a heterogeneous catalyst by strong adsorption of a particular component of the reaction system. Such poisons may be trace impurities in the feed, or may be a particular reaction (by-)product produced during the catalytic reaction. Although the precise mechanism of poisoning is dependent on the reaction undergoing study, the exact nature of the poison, the reaction conditions, and the active site speciation of the heterogeneous catalyst, it generally occurs through the strong $c f$. irreversible, chemisorption of the poison to the active site, minimising its ability to bind further reactant molecules. This can occur through physical or chemical processes. It is important to note that in addition to decreasing lifetime by progressively deactivating the catalyst, poisoning can also result in abrupt decreases in catalytic rates, and can therefore lead to decreased activity even during batch reactions. For this reason, intrinsic kinetic data should always be obtained at the initial stages of the reaction, to minimise the influence of product adsorption on the rate constants determined (Vide Supra).

The propensity for reaction species to act as a poison can be experimentally determined through intrinsic kinetic analysis. Comparison of the rate of reaction in the absence and presence of various contaminants can readily reveal how the rate of the catalytic reaction is modified by the presence of particular (by)products or impurities. A key element of this kinetic study is that the initial, i.e. intrinsic, rate of reaction is monitored, so as to minimise the impact that species produced during the standard reaction can also make to the reaction rate. Typically, the inverse rate constant is plotted as a function of the amount of potential poison added, so that a susceptibility factor can be calculated. Our group recently used this approach to investigate how various (by-)products impacted the rate of Baeyer-Villiger oxidation over $\mathrm{Sn}-\beta$. In this case, 6-hydroxyhexanoic acid (6$\mathrm{HHA}$ ), formed by hydrolysis of the primary lactone product (Figure 14), and water, formed through the use of $\mathrm{H}_{2} \mathrm{O}_{2}$ as oxidant, were found to decrease the initial rate of activity by up to $50 \%$, demonstrating their strong ability to act as poisons (Figure 15). 58

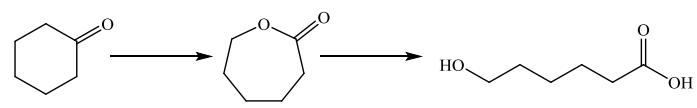

Figure 14. Formation of caprolactone and 6-hydroyexanoic acid through BVO and hydrolysis.

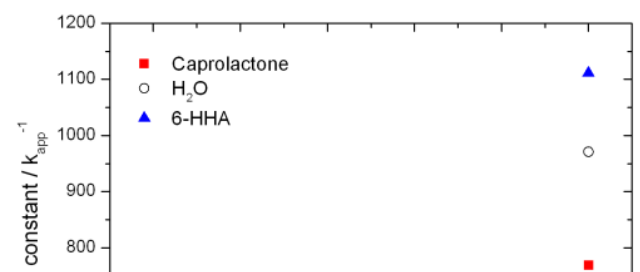


Figure 15. Effect of by-product addition on the initial rate of BVO over Sn- $\beta$. Reproduced with permission from reference 58 .

Extraction studies are also useful methods of identifying poisoning. In this case, treatment of the post reaction catalyst in various solvents, followed by ${ }^{1} \mathrm{H} /{ }^{13} \mathrm{C}$ (or related) NMR or chromatographic analysis of the supernatant solution, allows one to identify the presence of certain retained species. Through this manner, Mannel et al. identified that the deactivation of $\mathrm{Ru}(\mathrm{OH})_{\times} / \mathrm{Al}_{2} \mathrm{O}_{3}$ during the continuous oxidation of benzyl alcohol in toluene occurs primarily through the formation of benzoic acid. ${ }^{59}$ Indeed, the amount of benzoic acid removed from the catalyst post reaction upon treatment in aqueous $\mathrm{NaOH}$ correlated closely with the loss of activity $(0.90$ mol benzoate / $\mathrm{mol} \mathrm{Ru}$ ), strongly suggesting that one benzoate group binds to each Ru centre, resulting in deactivation of the metal site. ${ }^{59}$

Depending on the source of the particular poison, i.e. whether the poison is feed-based or produced during the reaction, mitigation strategies differ widely. For feed-based poisons, pre-treatment of the reaction solution prior to catalysis may be essential. ${ }^{62-65}$ For example, the removal of sulphur and analogous species from the feed is already widely followed in petrochemical processing, given the ability of such species to irreversibly poison metal-based catalysts. Similarly, the presence of particular solubilised cations e.g. $\mathrm{Na}^{+}, \mathrm{K}^{+}, \mathrm{Ca}^{2+}$, can readily poison, or even remove $\mathrm{Br} \varnothing n s t e d$ acid sites, through ionexchange. In this case, pre-treatment of the feed with ionexchange materials to remove such contaminants may be followed. ${ }^{60,61}$ It is often overlooked that several liquid phase feedstock are also obtained through acid- or base-catalysed depolymerisation processes. The presence of such contaminants can easily deactivate particular acid-base catalysts. Accordingly, upstream neutralisation of the feed may be required.

Water can also act as a poison, particularly to Lewis acid catalysts. Indeed, deactivation through hydrolysis is a wellknown limitation of several Lewis acid catalysts, including heterogeneous analogues. ${ }^{66}$ For example, the intrinsic activity of Sn- $\beta$ for the MPV transfer hydrogenation of cyclohexanone in 2-butanol decreased by more than $80 \%$ following the addition of only 4 wt. \% water to the feed. ${ }^{67}$ Furthermore, its ability to catalyse the isomerisation of glucose to fructose decreases by two orders of magnitude when water is employed as reaction solvent instead of methanol. ${ }^{68}$ In these cases, postsynthetic silylation of the zeolite material to increase its hydrophobicity, ${ }^{69}$ or modification of the reaction solvent resulted in improved rates of activity. Theoretical studies also indicate that different Lewis acids, such as $\mathrm{Zr}^{\mathrm{IV}}$, may be more resistant to poisoning by water. ${ }^{70}$ However, stability under continuous conditions was not evaluated in these previous studies.

Unfortunately, mitigation of catalytically produced poisons is far more challenging. Once particular (by-)products have been screened for poisoning ability, one may re-evaluate the performance of various catalysts to identify a candidate which possesses lower selectivity to the particularly undesirable compound(s). One may also consider putting particular additives into the reaction solution in order to minimise the production of a particular (by-)product. For example, Hammond et al. identified that the formation of trace amounts of benzoic acid was responsible for the deactivation of supported iridium oxide nanoparticles during the aerobic oxidation of benzyl alcohol. ${ }^{71}$ In this case, the formation of benzoic acid occured through radical based autoxidation. Accordingly, addition of trace amounts of a radical scavenger (2,6-di-tert-butyl- $p$-cresol) to the reaction solution halted the production of benzoic acid, and diminished the poisoning events observed. Additives such as $\mathrm{NaOH}$ can also minimise poisoning - and potentially, leaching - induced by chelating agents such as free carboxylic acids in solution. ${ }^{72,73}$

When the design of alternative catalytic materials possessing more suitable levels of selectivity is not possible, or the particular (by-product) responsible for deactivation cannot be scavenged or passivated, one can also mitigate poisoning by tuning the precise reaction conditions so that the production of the poison is minimised. For example, we recently identified that the major reason behind catalyst deactivation during the BVO of cyclohexanone over Sn- $\beta$ was the formation of the byproduct, 6 -HHA. ${ }^{58}$ Whilst catalyst- and process-optimisation studies demonstrated the hydrolysis reaction to be unavoidable i.e. the same active sites active for BVO were responsible for lactone hydrolysis, tuning the reaction conditions so that 6-HHA formation was less pronounced resulted in a more stable catalytic system. Indeed, operating the system so that reactant conversion was held below a conversion level of $60 \%$ meant that levels of 6-HHA were kept below a yield of $25 \%$ yield, at which point only a minor decrease in catalytic rate was observed. This resulted in a catalytic system displaying excellent levels of catalyst stability over a period of $200 \mathrm{~h}$. During this time period, $>5500$ substrate turnovers were obtained, a factor of 15 higher than previously obtained in any recyclability studies in batch reactors for the same catalytic system.

The same study also highlights the importance of measuring selectivity as a function of conversion (Figure 16, Right). As was observed by the authors, the unavoidable hydrolysis of caprolactone to 6-HHA resulted in increasingly lower levels of lactone selectivity as conversion increased. Accordingly, comparing the selectivity of different catalysts at different levels of conversion could lead to erroneous conclusions being made. To overcome this, the authors always compared lactone 
selectivity against ketone conversion (Figure 16, Right), which is one of the few methods of truly assessing reaction selectivity. This comparison revealed that optimizing the reaction conditions could result in significant improvements in lactone selectivity at a particular level of conversion; Indeed, lactone selectivity improved from $72 \%$ to $84 \%$ at $40 \%$ ketone conversion by optimizing the quantity of oxidant and water in the reactor. Moreover, the same optimization methods improved the $\mathrm{H}_{2} \mathrm{O}_{2}$-based selectivity (measured in terms of ketone converted / $\mathrm{H}_{2} \mathrm{O}_{2}$ converted $\mathrm{x} 100(\%)$ ) by a factor of 2, substantially improving the sustainability and economics of the system.
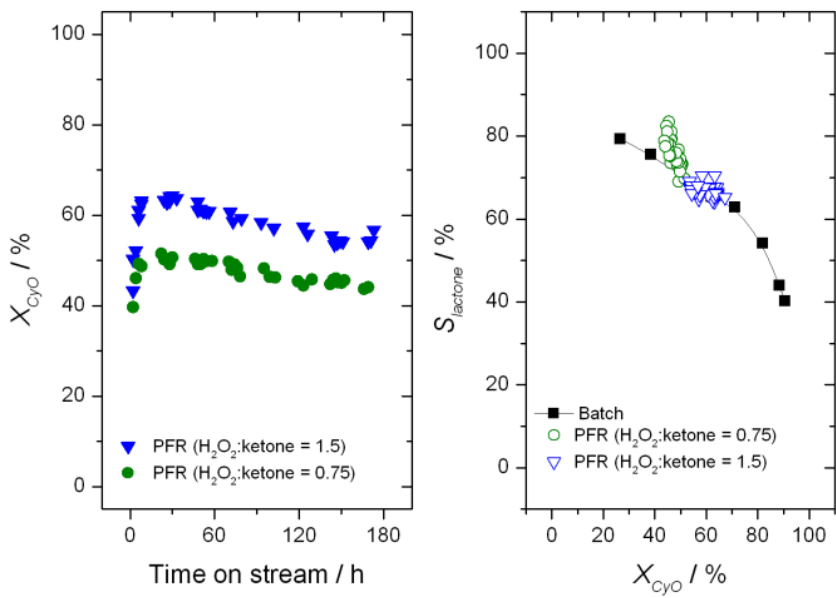

Figure 16. Extended time on stream performance of $\mathrm{Sn}-\beta$ during the Baeyer-Villige oxidation of cyclohexanone at $100{ }^{\circ} \mathrm{C}$. (Left) Cyclohexanone conversion as a function of time on stream, and (Right) Lactone selectivity as a function of ketone conversion. Reproduced with permission from reference 58

3.4 Active site reorganisation. The reorganisation of active sites during catalysis typically leads to deactivation through a decreased number of active sites. This can occur through several distinct methods, including (1) restructuring leading to a decrease in the accessible surface area of the catalyst i.e. sintering, (2) complete loss of an active species through formation of a new, inactive species, or (3) loss of the active site through leaching. Such processes can be catastrophic e.g. restructuring into an inactive site or leaching, or they may subtler, such as the change in ligand environment modifying the activity and/or selectivity of an active site.

Reorganisation may be related to the catalytic reaction (a particular by-product or contaminant resulting in reorganisation of the active site), or may solely be due to the process conditions themselves i.e. (solvo)thermal modification of the active sites, with little influence from the reaction. Given that sintering is strongly temperature dependent, ${ }^{21}$ it may be anticipated that its effects are less severe for heterogeneous catalysts mediating liquid phase processes, given that such processes are characterised by milder conditions than gasphase analogous. However, the presence of a solvent can markedly affect the stability, and hence reorganisation, of particular active sites. ${ }^{39}$ Indeed, this facet is one of the least known elements of heterogeneous catalysts operating in the liquid phase. Whilst active site reorganisation may be a precursor to leaching (see 3.5), these events may also be independent of one another.
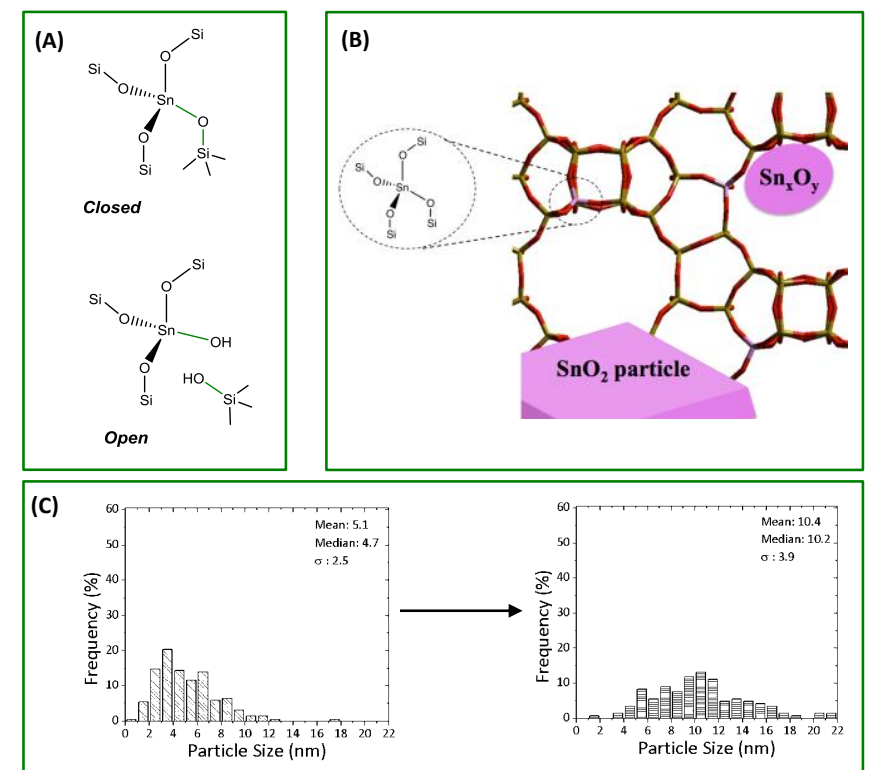

Figure 17. A variety of restructuring events that may lead to catalyst deactivation, including (A) hydrolysis and alkoxylation of isomorphously substituted heteroatoms, (B) the migration of isomorphously substituted heteroatoms into inactive oligomeric or oxidic forms, and (C) metal nanoparticle sintering following catalytic operation.

Active site reorganisation can occur through several different methods depending on the composition of the catalyst. For example, reorganisation of isomorphously substituted heteroatoms within zeolite frameworks to (inactive) extra-framework oligomers, or even bulk oxide particles, is a process that can easily result in diminished levels of activity (Figure 17). Likewise, changes to the direct coordination sphere of the isomorphously substituted heteroatoms through hydrolysis or alkoxylation can also occur. Another type of reorganisation includes sintering of metal nanoparticles into larger agglomerates, characterised by decreased intrinsic activity and also a diminished accessible surface area.

Accordingly, determining the type and extent of active site reorganisation relies extensively on detailed characterisation of the active site prior to, and following, continuous operation. A variety of methods including Raman, UV-Vis and FTIR spectroscopy, Microscopy (TEM, STEM), X-ray Absorption Spectroscopy (XAS), NMR ((DNP-)MAS-NMR) Thermal methods (TPDRO) and Mössbauer, can be utilised. ${ }^{74-80}$ For example, Lari et al. employed a combination of UV-Vis spectroscopy and DRIFT studies with $\mathrm{d}^{3}$-deuterated $\mathrm{CD}_{3} \mathrm{CN}$ probe molecules, to determine the extent of reorganization of stannosilicate zeolites during the isomerization of dihydroxyacetone and xylose in water or methanol.81 Depending on the reaction undergoing study and the choice of catalyst, reorganization occurred either through clustering of $\mathrm{Sn}^{\mathrm{IV}}$ into extra-framework SnOx species, or extensive reorganization into bulk $\mathrm{SnO}_{2}$ on the external surface of the zeolite (Figure 17). Wang et al. also employed DRIFT studies with $\mathrm{d}^{3}$-deuterated $\mathrm{CD}_{3} \mathrm{CN}$ probe 
molecules in order to the deactivation of $\mathrm{Hf}-\beta$ zeolites for the aldol condensation of ethyl puryvate.

Our group recently employed a combination of X-ray Absorption spectroscopy (XANES, EXAFS) and ${ }^{119}$ Sn MAS NMR in order to probe the reorganisation of $\mathrm{Sn}-\beta$ during catalytic transfer hydrogenation in 2-butanol (Figure 18). ${ }^{36}$ In this case, extraction of isomorphously substituted $\mathrm{Sn}^{\mathrm{IV}}$ was also observed. However, the extent of active site reorganisation was substantially less severe than observed by Lari et al. The disparate degrees of reorganisation demonstrate how the precise reaction conditions (solvent, temperature etc.) can strongly influence the rate and degree of reorganisation.

Subtle active site reorganization of $\mathrm{Sn}-\beta$ was also detected by Lewis et al. during the transfer hydrogenation of 5hydroxymethyl furfural. There, ex reactor analysis of Sn- $\beta$ by ${ }^{119} \mathrm{Sn}$ MAS NMR was performed. In this case, the changes in active site speciation were subtler, and were attributed to changes in the coordination environment of the active site, in terms of ligands and geometry.
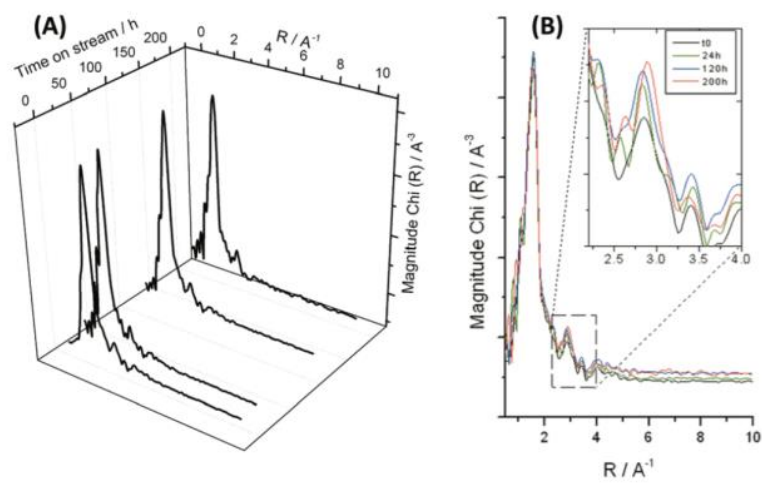

Figure 18. X-ray Absorption spectroscopy analysis of $\mathrm{Sn}-\beta$ during continuous transfer hydrogenation catalysis in 2-butanol at $100{ }^{\circ} \mathrm{C}$. Reproduced with permission from reference 36 .

For supported metal nanoparticles, the primary mode of reorganization involves sintering. Since available surface area is directly related to the particle size, such events drastically reduce the number of surface atoms available for reaction. Moreover, they may also reduce the ability of each metal site to perform the intrinsic reaction, particularly if there is a strong dependence of activity on particle size. ${ }^{83}$ Microscopic methods such as (S)TEM, ${ }^{84}$ can readily demonstrate that active site reorganization occurs. Restructuring of nanoparticles of AuPd has also been studied by combined EXAFS/DRIFTS, 85 and (pulse) chemisorption studies ${ }^{86}$ can also be used to readily identify sintering events. Where reorganisation is related to the oxidation or reduction of a particular phase, e.g. the formation of $\mathrm{Pd}^{2+}$ during the reaction, techniques such as TPDRO, XRD and $X P S$ can provide useful information. Information on the formation, or conversion of, particular metal oxides phases can also be detected through vibrational methods such as Raman, and PXRD.

Depending on the nature of the catalyst and the extent of active site reorganization, methods of minimizing structural reorganization differ widely, although they can generally be placed into two broad categories; (1) catalyst design modifications, and ( 2 ) reaction environment modifications.

Where reorganization is related to the sintering or agglomeration, the metal loading of the catalyst can be lowered to increase site isolation. For example, we recently demonstrated that the formation of oligomeric SnOx phases in Sn- $\beta$ is strongly related to the metal loading, with increased loadings above $5 \mathrm{wt}$. \% Sn leading to substantial increases in agglomeration, as detected by XAS (Figure 19) and ${ }^{119}$ Sn MAS NMR spectroscopy. ${ }^{57}$ The formation of such phases was accompanied by a large decrease in TOF. Whilst lowering the active site concentration leads to unwanted decreases in catalyst productivity, a balance between activity and stability is required.

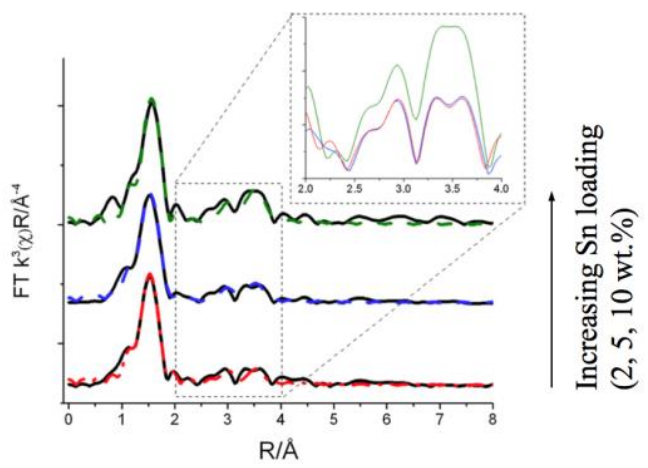

Figure 19. X-ray Absorption spectroscopy analysis of $\mathrm{Sn}-\beta$ as a function of Sn loading. Increasing the $\mathrm{Sn}$ loading above $5 \mathrm{wt}$. \% Sn results in an increase in $\mathrm{Sn}$-Sn interactions due to the formation of extra-framework $\mathrm{Sn}$ species. Adapted with permission from reference 57

For zeolites and analogous porous frameworks, encapsulation of the same active site within a different framework can strongly impact activity and stability. Indeed, it is well known that different frameworks exhibit different degrees of stability, both in terms of resistance to hydrolysis/dissolution (see 3.6) and also in terms of active site reorganisation. For example, Lari et al. recently demonstrated that the reorganisation of isomorphously substituted $\mathrm{Sn}^{\mathrm{IV}}$ atoms was significantly greater in Sn-MOR than Sn-MFI during continuous sugar isomerisation. ${ }^{81}$

The sintering of metal nanoparticles is strongly dependent on (1) the choice of support, (2) the initial particle size, (3) the composition of the metal nanoparticle (monometallic or multiimetallic, alloy or coreshell), (4) the method of deposition, and (5) the presence of particular stabilisers. These factors are widely discussed in several excellent reviews, and therefore will not be overemphasised here. ${ }^{87-89}$ However, changing the support material, employing a different preparation procedure, alloying the metal and modifying the preparation procedure can drastically improve resistance to sintering.

A key stage during catalyst synthesis is the final pretreatment process, typically performed at elevated temperatures. Optimisation of this process can also result in the formation of more stable active site phases. Indeed, $\mathrm{TiCl}_{4}-$ grafted silica was shown to be a more active and stable epoxidation catalyst when pre-treated at $450{ }^{\circ} \mathrm{C}$ instead of 250 
${ }^{\circ} \mathrm{C} .{ }^{90}$ Increased stability in this case was attributed to a greater interaction between the grafted Tilv centres and the silica support following the higher temperature treatment (Figure 20).

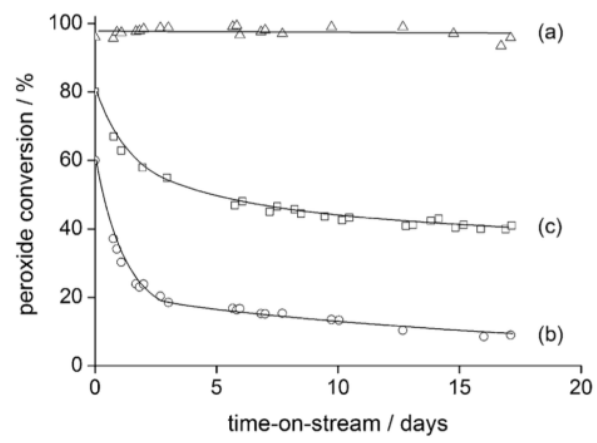

Figure 20. Activity and stability of various silica-grafted $\mathrm{TiCl} 4$ catalysts during the solventfree epoxidation of cyclooctene. Catalyst (a) was pre-treated at $450{ }^{\circ} \mathrm{C}$, catalyst (b) was pre-treated at $250^{\circ} \mathrm{C}$. A Ti $\left(\mathrm{O}^{\prime} \mathrm{Pr}\right)_{4}$-impregnated catalyst (c) is also compared. Reproduced with permission from reference 90 .

If the catalyst composition cannot be finely tuned to provide sufficiently stability, then analogous active sites of a different composition may also be considered. For example, Lewis et al. demonstrated through MAS NMR spectroscopy that for a given number of TON, Sn- $\beta$ deactivates more gradually than its $\mathrm{Hf}$ containing analogue during the transfer hydrogenation and etherification of 5-hydroxymethyl furfural. 82 For a similar reaction (Figure 21), we also demonstrated that adding of a small amount of Brønsted acidity to a Sn-containing zeolite led to substantial improvements in stability of the transfer hydrogenation/etherification system. ${ }^{91}$ In this case of bifunctional Sn,Al- $\beta$, BMF selectivity was maintained above 70 $\%$ even after 2300 turnovers, whereas the selectivity in the Snonly system decreased to $<30 \%$ after only 900 turnovers (Figure 22).

Additional treatments of the heterogeneous catalyst can also be followed. Where active site reorganization is related to the interaction with a particular reaction product or solvent (Vide Infra), then treatment of the catalyst to minimize such interactions may be feasible. A key example of this is silylation. ${ }^{69}$ Overcoating of single layers of a suitable metal oxide, or carbon, onto the surface of a catalyst by Atomic Layer Deposition has also been found to improve active site stability. ${ }^{92}$ These improvements have been attributed to the preferential coating of the most undercoordinated, and hence most exposed, metal sites, which likely provide the most suitable initiators of sintering. However, such treatments may also coat the most active surface sites, leading to a compromise between activity and stability. This topic was recently reviewed by Heroguel et al. ${ }^{93}$

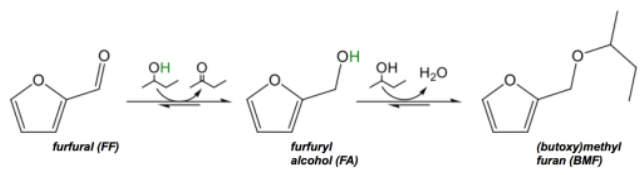

Figure 21 Catalytic formation of furanic ethers from furfural.

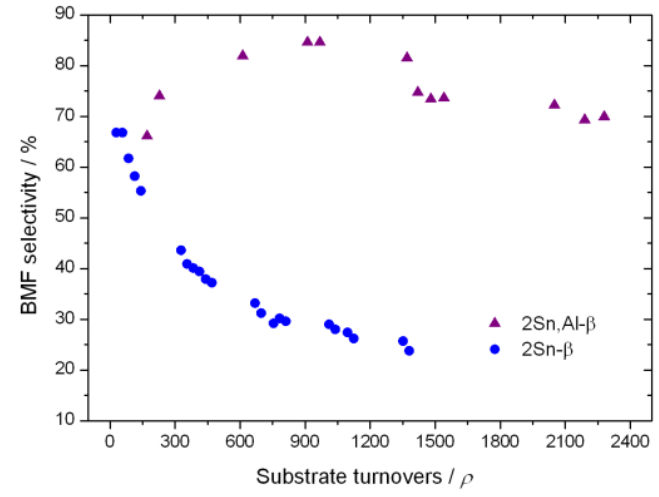

Figure 22. BMF selectivity obtained during transfer hydrogenation/etherification of furfural with $\mathrm{Sn}$ - and $\mathrm{Sn}, \mathrm{Al}$-containing $\beta$ zeolite. Data submitted for publication.

These studies also highlight an important aspect that should always be remembered of heterogeneous catalysts, which is that a single active site or species is rarely present prior to catalysis, and especially during the catalytic reaction itself. Typically, a variety of sites and species are present, each of which may contribute differently to the overall performance of the catalyst, and which may (inter)convert into one another particularly under the reaction conditions. Accordingly, the characterisation of such materials, particularly during continuous operation, is highly challenging, and will benefit greatly from improvements in the area of in situ spectroscopy (see section 4 for further discussion).

3.5 Leaching. Leaching describes the solubilisation of particular components of the catalytic material into the reaction medium. A form of irreversible deactivation (in a continuous system the solubilised species are flushed out of the reactor), leaching can have significant consequences for a process; The presence of potentially toxic species in solution - particularly heavy metals - can damage the environmental feasibility of the process. Moreover, the loss of expensive catalytic elements, coupled with increased downstream processing requirements for recovery of the leached elements and decontamination of the effluent, can significantly damage the economic potential of a process. Accordingly, avoiding leaching is critical. Metal leaching, especially during liquid phase biomass upgrading, was recently reviewed by Sadaba et al., and consequently this specific element of catalyst deactivation will not be covered as extensively as the others in this review, although it is included briefly for completeness.

Leaching can be detected by analysis of the liquid phase of the reaction, the residual solid phase after reaction, or ideally, both. Elemental analysis methods, such as ICP-MS or AES are most suitable. The liquid phase of the reaction is typically sampled at reaction conditions, and subsequently analysed for the presence of the potential leached components. Sampling a reaction solution following a batch reaction at low temperature can be misleading, as re-deposition of the active element onto the solid can occur. However, such issues are not observed for 
continuous experiments, as the leached species are continually washed away from the solid. Although elemental analysis techniques do provide high degrees of detectability and element specificity, it is critical to note that the precise detectability limit is technique, element and sample dependent. Moreover, for catalysts containing only a small weight percentage of active sites, even moderate amounts of leaching may not lead to sufficient concentration of the leached species in the effluent for detectability to be observed. For these reasons, hot filtration experiments, as outlined above (2.1) are essential to rule out the potential activity of leached metal species in batch systems.

To further determine potential leaching events, one can also verify the active site concentration of the fresh and used catalytic material with elemental analysis methods. To achieve this, the solid catalyst can either be digested in suitable media e.g. aqua regia or $\mathrm{HF}$, and subsequently analysed by typical liquid phase methods as described above, or alternative elemental analysis methods capable of directly studying solid materials, such as XRF, EDX, or even XPS or UV-Vis spectroscopy, can be employed. Given that several of these alternative methods are only semi-quantitative at best, particularly if the material has a non-homogeneous distribution of active sites between surface and bulk, then digestion methods are always preferred, provided full digestion can be achieved. Indeed, poor methods of digestion e.g. incomplete dissolution of components of the material, can strongly influence the obtained results. Regardless of the approach employed, then leaching is often reported as a percentage of active site in solution as a function of the original metal loading.

Broadly speaking, leaching can also be considered a type of active site reorganisation (see 3.5 ). In the case of leaching, however, an immobilised phase of the solid becomes a new, soluble phase, which can subsequently be dissolved into the reaction media upon formation. Whilst the consequences of leaching can be far more severe, the strategies for the mitigation of active site reorganisation and leaching are actually very similar, since leaching is a consequence of poor active site stability. Accordingly, all the mitigation methods described in 3.4 can be explored. As these are described in depth above, they will not be repeated here.

Where only part of the active site is susceptible to leaching, then pre-leaching the unstable component by a pre-treatment in a suitable solvent medium may be performed. Although this still classifies as leaching, pre-leaching the unstable component under controlled conditions prior to catalysis minimises the opportunity for leaching to impact the overall catalytic process, contaminate downstream reactors and products, and may result in the leached species being recovered more readily. ${ }^{95}$

3.6 Hydrothermal dissolution. Deactivation through hydrothermal dissolution is related to the destruction or dissolution of the catalyst through interaction of the catalyst with the hot solvent. For Lewis acidic silicates, such dissolution may relate to loss of the crystalline structure and hence, total reorganisation of the active sites. For other heterogeneous catalysts, such dissolution may be related to the formation of new phases of a metal/metal oxide, or restructuring of the active sites e.g. sintering, upon treatment in the hot liquid medium. Such processes may be gradual or rapid, and may result in partial or total destruction of the catalyst.

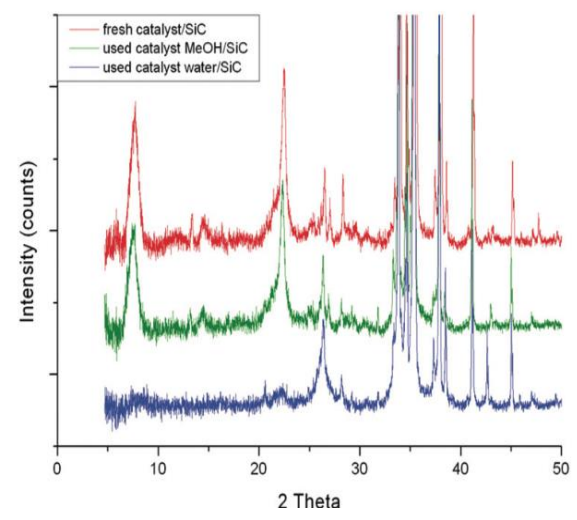

Figure 23. Loss of crystallinity exhibited by $\mathrm{Sn}-\beta$ during aqueous phase glucose isomerisation. The now-armosphous material (blue/bottom) is compared to a sample of the fresh catalyst (red/top) and a catalyst that performed the reaction in methanol solvent (green/middle). Reproduced with permission from reference 36 .

Hydrothermal dissolution of the catalyst is best identified through spectroscopic characterisation of the catalytic material prior to, and following, continuous operation. For crystalline materials, powder XRD ( $P X R D$ ) may provide suitable insight into hydrothermal dissolution of the catalyst. For example, we recently employed pXRD to demonstrate that amorphisation of $\mathrm{Sn}-\beta$ occurs during the aqueous phase isomersation of glucose to fructose at $110{ }^{\circ} \mathrm{C}$ (Figure 23). ${ }^{36}$ However, it should be noted that pXRD alone may not be sufficient to detect partial amorphisation. Indeed, partial pore collapse may be more readily identified by micropore volume measurements (porosimetry), particularly after potential fouling has been remedied by regeneration. For example, Van der Vyver only detected partial degradation of $\mathrm{Sn}-\beta$ by performing detailed micropore volume measurements; Although such volumes decreased by $\pm 20 \%$, strongly indicating at least some structural degradation, no evident changes to the crystalline structure could be detected by pXRD. ${ }^{96}$

Modifying the reaction solvent, and choosing the optimal solvent both in terms of activity and stability, represents one of the major mitigation strategies. Indeed, the precise properties of the solvent can intimately impact both reversible (fouling) and irreversible (hydrothermal dissolution) causes of catalyst deactivation. For example, despite its reported watertolerance, the stability of $\mathrm{Sn}-\beta$ during the isomerisation of glucose to fructose is substantially higher when methanol is employed as reaction solvent; In water, extensive and irreversible deactivation due to amorphisation of the zeolite was observed by pXRD and porosimetry (Figures 23-25). ${ }^{36}$ In contrast, performing the reaction in methanol leads to a shift in deactivation mechanism, with fouling being the primary reason for decreased in activity. Lari et al. also observed similar results whilst investigating the isomerisation of dihydroxyacetone and xylose over Sn-MFI, Sn-MOR, Sn-BEA and Sn-FAU zeolites. ${ }^{81}$

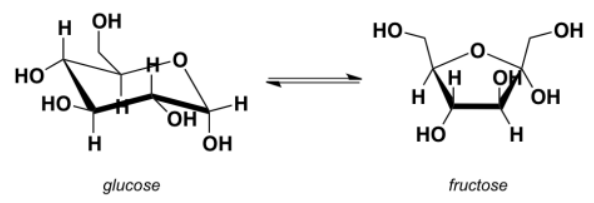




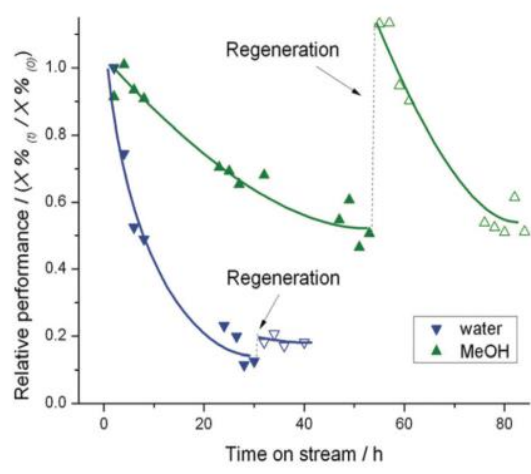

Figure 25. Influence of reaction solvent on the stability of $\mathrm{Sn}-\beta$ during the isomerisation of glucose to fructose. Reproduced with permission from reference 36 .

Even use of a co-solvent, whether miscible or immiscible, may also lead to increased catalyst stability. Indeed, Aellig and colleagues observed that an immiscible solvent mixture of water $(20 \%)$ and methyl isobutyl ketone ( $80 \%)$ resulted in significant improvements in the stability of a bifunctional catalyst bed comprising of a physical mixture of Ga-containing USY and Amberlyst-36, during the catalytic isomerisation and dehydration of xylose. ${ }^{97}$ In this case, an immiscible Taylor flow of the two co-solvents proved more suitable than an aqueous solvent alone, and resulted in decreased metal leaching and increased stability of the catalyst. Reportedly, this improvement was due to the organic co-solvent minimising the negative interaction of water with the catalyst, which was responsible for deactivation. In addition, the co-solvent also resulted in improved levels of target product selectivity by extracting the relatively unstable reaction product, furfural, from the aqueous phase, preventing its subsequent hydrolysis.

Unfortunately, bio-renewable feedstocks are rarely available in the complete absence of water. Moreover, the most economically viable sugar feedstock is sugar syrup, which possesses a high content of water. Accordingly, in light of the negative role played by water in these systems, effort has also been undertaken to identify which matrices and frameworks offer greatest water tolerance. It has been observed hydrothermally-prepared $\mathrm{Sn}-\beta$ is more resistant to hydrolysis than post-synthetically prepared analogues, and thus exhibits more favourable stability properties when reactions are performed in the aqueous phase. ${ }^{98}$ Reportedly, this is due to the increased hydrophobicity of the defect-free framework, which is observed when the zeolite is crystallised in a fluoride media. However, even for such materials deactivation in an aqueous medium is still observed. In a similar approach, post-synthetic silylation of zeolites with organosilanes has also been attempted, in order to improve their hydrophobicity and consequently minimise the hydrolysis of their active centres. ${ }^{69}$
Given the vast differences exhibited by different alumionsilicate frameworks for hydrothermal dissolution, changing the overall framework composition to one more resistant to hydrolysis may also lead to significant improvements in activity and stability. ${ }^{99}$ Improved hydrothermal stability has also been achieved by overcoating with carbon ${ }^{100}$ (see 3.4 ), and it should be added that other classes of catalytic material may be less susceptible than Lewis acidic zeolites to degradation in the aqueous phase.

\section{Conclusions, perspectives and pertaining challenges}

Successfully commercialising a new catalytic process intimately depends on the development of novel heterogeneous catalysts possessing not only high levels of activity and selectivity, but also sufficient levels of stability. Consequently, studying and mitigating deactivation, especially for LS processes, is essential.

Several potential processes can intimately impact the stability of a catalytic material, and many of these events can act in parallel. However, promising breakthroughs with regards to elucidation of deactivation phenomena, and strategies for their mitigation, are being made. It should be stressed that whilst these methods may not be able to completely overcome catalyst deactivation i.e. some deactivation will always occur, if they are able to mitigate it for a sufficient period of time then a potential process may still become viable from an economic perspective to be performed.

With this in mind, several challenges remain to be tackled, and should form the basis of future research studies in this field. Firstly, it is readily apparent from the literature that most studies focused on catalyst deactivation do not probe the deactivation events deeply enough for sufficient stability information to be obtained. Accordingly, a major challenge in this area is for the stability studies, as performed in an academic environment, to be truly relatable to those required by the chemical industry. Thus, despite the usefulness of recyclability studies as a preliminary insight into catalyst stability, more emphasis needs to be placed on hot filtration experiments and/or complimentary leaching studies, ${ }^{101}$ and ideally, continuous technologies. In the literature, it is notable that "extended" time on stream studies are often performed for an inadequate amount of time, and/or low reactant flow rates are employed. Consequently, the TON or productivity values exhibited by the catalyst can often be the same, or in some cases, lower than is achieved during recyclability studies in batch reactors. As such, continuous stability studies should always be performed for a significant period of time to allow sufficient turnover and deactivation to occur. To aid this research, accelerated ageing methodologies can also be developed.

Secondly, whilst continuous methods are more suitable, they only become truly informative when paired with complimentary spectroscopic study. This review has demonstrated that coupling simple, laboratory based methods e.g. UV-Vis, Raman, XRD, porosimetry, with advanced 
spectroscopic measurements, e.g. XAS, that a substantial amount of information concerning the nature of the deactivation events occurring during liquid phase operation. However, it should be remembered that the true active site of a catalyst only exists in the catalytic reactor, ${ }^{102}$ and hence preand post-reaction characterisation may not provide the best levels of insight as they may, in extreme cases, totally miss the real site of interest. For these reasons, efforts should also be focused upon the development of new reactor environments, or new spectroscopic methods, capable of probing solid catalysts in a liquid phase environment (in situ spectroscopy). ${ }^{103}$ In fact, whilst the field of in situ spectroscopy has rapidly emerged over the last decade, most in situ methods have been optimised for the study of heterogeneous catalysts mediating gas-solid processes. Hence, catalysis researchers focused on liquid phase processing do not yet have access to suitable number tools for studying the active sites without potentially destroying them by removing them from the reactor, which seriously limits detailed spectroscopic study. It should be added that a major advantage of continuous reactors in a spectroscopic sense is that they operate under steady state conditions. As such, performance of the catalyst along the reactor axis is not just a function of time but also a function of its bed position. This allows one to monitor the catalytic system both by monitoring a fixed bed position whilst varying the reagent flow rates and thus the contact time (conventional method), or alternatively by maintaining reactant flow rates and varying the bed position monitored, i.e. by mapping the catalyst bed. The second approach can lead to improved levels of time resolution, can provide information on non-linear effects, and also allows one to readily examine the performance of the catalyst as a function of bed length, which is particularly important given that each catalyst particle along the reactor axis is exposed to a different ratio of reactants and products. ${ }^{104-}$ 107

In addition to these overarching challenges, several smaller hurdles also require study. The first is the development of alternative regeneration protocols. Indeed, whilst typical regeneration methods rely on high-temperature treatment of a deactivated solid catalyst, such protocols have been optimised based on established GS processes, and may not be optimal for emerging heterogeneous catalysts, particularly if they possess thermally unstable components. Moreover, they also require a LS reactor to be drained, and have provision for high temperature heating. Potential alternatives include solvent washing and/or extraction, or milder temperature regenerations.

Additionally, it is clear that the design of hydrothermally stable materials is critical. Indeed, even if good active site stability can be achieved, dissolution of the support can still result in deactivation of a heterogeneous catalyst.

Whilst water may be unavoidable during biomass upgrading, it does not necessarily represent the 'green' solvent it is often portrayed as. Indeed, in addition to increasing rates of dissolution, leaching and deactivation, the remediation of toxic species and by-products from wastewater represents a costly and difficult challenge. Accordingly, the employment of alternative solvents should still be explored.

On a final, related note, we also briefly mention that utilisation of a heterogeneous catalyst on an industrial scale also requires intensification of the catalyst synthesis procedure. However, most studies to date have not truly addressed the issue of catalyst synthesis scalability, with catalyst preparation still being performed on the gram scale. Although not related to catalyst deactivation, it relates to the concept of scalability, and should also receive addition focus in the coming years.

\section{Acknowledgements}

$\mathrm{CH}$ gratefully appreciates the support of The Royal Society, for provision of a University Research Fellowship (UF140207) and further research grant funding ( $\mathrm{CH} 140207)$.

\section{References}

1 F. Cavani, J. H. Teles, ChemSusChem 2009, 2, 508

2 F. Cavani, Catal. Today. 2010, 157, 8.

3 J. M. Thomas, R. Raja, D. W. Lewis, Angew. Chem. Int. Ed. 2005, 44, 6456;

4 A. Corma, H. Garcia, Chem. Rev. 2003, 103, 4307-4365.

5 A. Corma, S. Iborra, A. Velty, Chem. Rev. 2007, 107, 2411.

6 P. N. R. Vennestrøm, C. M. Osmundsen, C. H. Christensen, E. Taarning, Angew. Chem. Int. Ed. 2011, 50, 10502.

7 J. S. Luterbacher, D. Martin Alonso, J. A. Dumesic, Green Chem. 2014, 16, 4816.

8 E. Taarning, C. M. Osmundsen, X. Yang, B. Voss, S. I. Andersen, C. H. Christensen, Energy Environ. Sci. 2011, 4, 793.

9 R. Rinaldi, F. Schüth, ChemSusChem 2009, 2, 1096.

10 X. Zhang, K. Wilson, A. F. Lee, Chem. Rev. 2016, DOI: 10.1021/acs.chemrev.6b00311

11 P. Lanzafame, D. M. Temi, S. Perathoner, A. N. Spadaro, G. Centi, Catal. Today 2012, 179, 178.

12 I. Delidovich, K. Leonhard, R. Palkovits, Energy Environ. Sci., 2014, 7, 2803,

13 T. Ennaert, J. V. Aelst, J. Dijkmans, R. De Clercq, W. Schutyser, M. Dusselier, D. Verboekend, B. F. Sels, ChemSocRev. 2016, 45, 584.

14 C. O. Tuck, E. Pérez, I. T. Horváth, R. A. Sheldon, M. Poliakoff, Science, 2012, 337, 695.

15 R. A. Sheldon, Green Chem. 2014, 16, 950.

16 J.-P. Lange, CatTech 2001, 5, 82.

17 J.-P. Lange, Angew. Chem. Int. Ed. 2015, 54, 13186.

18 P. A. Jacobs, M. Dusselier, B. F. Sels, Angew. Chem. Int. Ed. 2014, 53, 8621.

19 C.-H. Zhou, X. Xia, C.-X. Lin, D.-S. Tong, J. Beltramini, Chem. Soc. Rev. 2011, 40, 5588.

20 T. J. Schwartz, B. J. O'Neill, B. H. Shanks, J. A. Dumesic, ACS Catal. 2014, 4, 2060.

21 C. H. Bartholomew, Appl. Catal. A: Gen. 2001, 212, 17.

22 I. W. C. E. Arends, R. A. Sheldon, Appl. Catal. A: Gen. 2001, 212, 175.

23 M. S. Holm, S. Saravanamurugan, E. Taarning, Science, 2010, 328, 602 .

24 M. Moliner, Y. Roman-Leshkov, M. E. Davis, Proc. Nat. Acad. Sci., 2010, 107, 6164.

25 C. Hammond, S. Conrad, I. Hermans, Angew. Chem. Int. Ed. 2012, 51, 11736.

26 A. Corma, L. T. Nemeth, M. Renz, S. Valencia, Nature 2001 412, 423. 
27 N. T. S. Phan, M. Van der Sluys, C. W. Jones, Adv. Synth. Catal. 2006, 348, 609.

28 R. A. Sheldon, M. Wallau, I. W. C. E. Arends, U. Schuchardt, Acc. Chem. Res. 1998, 31, 485.

29 L. Yin, J. Liebscher, Chem. Rev. 2007, 107, 133.

30 L. Forni, Catal. Today 1997, 34, 353.

31 R. L. Hartman, J. P. McMullen, K. F. Jensen, Angew. Chem. Int Ed. 2011, 50, 7502;

32 C. Perego, S. Peratello, Catal. Today 1999, 52, 133.

33 J. Peŕez-Ramırez, R. J. Berger, G. Mul, F. Kapteijn, J. A. Moulijn, Catal. Today 2000, 60, 93.

34 A. S. Piskun, J. E. de Haan, E. Wilbers, J. J. van de Bovenkamp, Z. Tang, H. J. Heeres, ACS Sust. Chem. Eng. 2016, 4, 2939.

35 R. J. Berger, J. Perez-Ramirez, F. Kapteijn, J. A. Moulijn, Chem. Eng. Sci. 2002, 57, 4921.

36 D. Padovan, C. Parsons, M. S. Grasina, C. Hammond, Green Chem., 2016, 18, 50413.

37 C. Aellig, I. Hermans, ChemSusChem 2012, 5, 1737.

38 J. A. Moulijn, A. E. van Diepen, F. Kapteijn, Appl. Catal. A: Gen. 2001, 212, 3.

39 I. Sadaba, M. Lopez Granados, A. Riisager, E. Taarning, Green Chem. 2015, 17, 4133.

40 P. Forzatti, L. Lietti, Catal. Today 1999, 52, 165.

41 L. W. Kroh, Food Chem. 1994, 51, 373.

42 M. Choura, N. M. Belgacem, A. Gandini, Macromolecules 1996, 29, 3839.

43 M. Koehle, R. F. Lobo, Catal. Sci. Tech. 2016, 6, 3018.

44 C. T. Campbell, Acc. Chem. Res. 2013, 46, 1712.

45 D. Martin Alonso, S. G. Wettstein, M. A. Mellmer, E. I. Gurbuzab, J. A. Dumesic, Energy Environ. Sci. 2013, 6, 76.

46 J. Jiang, J. Yu, A. Corma, Angew. Chem. Int. Ed. 2010, 49, 31203145

47 C. C. Freyhardt, M. Tsapatsis, R. F. Lobo, K. J. Balkus Jr., M. E. Davis, Nature, 2007, 391, 295.

48 A. Corma, M. J. Diaz-Cabañas, J. Martinez-Triguero, F. Rey, J. Rius, Nature, 2008, 418, 514.

49 M. Choi, K. Na, J. Kim, Y. Sakamoto, O. Terasaki, R. Ryoo, Nature, 2009, 461, 246.

50 K. Li, J. Valla, J. Garcia-Martinez, ChemCatChem, 2014, 6, 46.

51 J. Perez-Ramirez, C. H. Christensen, K. Egleblad, C. H. Christensen, J. C. Groen, Chem. Soc. Rev., 2008, 37, 2530.

52 M. Hartmann, Angew. Chem. Int. Ed., 2004, 43, 5880.

53 A. Al-Nayili, K. Yakabi, C. Hammond, J. Mater. Chem. A, 2016, 4, 1373

54 X. Ouyang, S.-J. Hwang, D. Xie, T. Rea, S. I. Zones, A. Katz, ACS Catal. 2015, 5, 3108.

55 H. Y. Luo, L. Bui, W. R. Gunther, E. Min, Y. Roman-Leshkov, ACS Catal. 2012, 2, 2695.

56 P. Y. Dapsens, C. Mondelli, J. Jagielski, R. Hauert,. PérezRamírez, Catal. Sci. Technol., 2014, 4, 2302.

57 C. Hammond, D. Padovan, A. Al-Nayili, P. P. Wells, E. K. Gibson, N. Dimitratos, ChemCatChem, 2015, 7, 3322.

58 K. Yakabi, K. Milne, A. Buchard, C. Hammond, ChemCatChem 2016, 6, 1-10.

59 D. S. Mannel, S. S. Stahl, T. W. Root, Org. Process. Res. Dev. 2014, 18, 1503.

60 P. S. Metkar, E. J. Till, D. R. Corbin, C. J. Pereira, K. W. Hutchenson, S. K. Sengupta, Green Chem. 2015, 17, 1453 1466.

61 Y. Takeshita, Y. Sato, S. Nishi, Ind. Eng. Chem. Res. 2000, 39, 4496.

62 B. Katryniok, S. Paul, V. Belliõre-Baca, P. Reye, F. Dumeignil, Green Chem. 2010, 12, 2079.

63 J. F. Saeman, Ind. Eng. Chem., 1945, 37, 43.

64 R. W. Torget, J. S. Kim and Y. Y. Lee, Ind. Eng. Chem. Res., 2000, 39, 2817.

65 Y.-B. Huang, Y. Fu, Green Chem. 2013, 15, 1095.

66 Y. Román-Leshkov, M. E. Davis, ACS Catal. 2011, 1, 1566.
67 A. Corma, M. E. Domine, S. Valencia, J. Catal. 2003, 215, 294. 68 R. Gounder, M. E. Davis, J. Catal. 2013, 308, 176.

69 P. A. Zapata, J. Faria, M. P. Ruiz, R. E. Jentoft, D. E. Resasco, J. Am. Chem. Soc. 2012, 134, 8570.

70 R. J. Assary, L. A. Curtiss, J. Phys. Chem. A. 2011, 115, 8754.

71 C. Hammond, M. T. Schümperli, S. Conrad, I. Hermans, ChemCatChem 2013, 5, 2983.

72 S. Biella, L. Prati, M. Rossi, J. Catal. 2002, 206, 242.

73 D. D. Perrin, J. Chem. Soc. 1958, 3125.

74 S. R. Bare, S. D. Kelly, W. Sinkler, J. J. Low, F. S. Mmodica, S. Valencia, A. Corma, L. T Nemeth, J. Am. Chem. Soc. 2005, 127, 12924.

75 P. Wolf, M. Valla, A. J. Rossini, A. Comas-Vives, F. Nunez-Zarur, Angew. Chem. Int. Ed. 2014, 126, 10343;

76 W. R. Gunther, V. K. Michaelis, M. A. Caporini, R. G. Griffin, Y. Roman-Leshkov, J. Am. Chem. Soc. 2014, 136, 6219.

77 Y. G. Kolyagin, A. V. Yakimov, S. Tolborg, P. N. R. Vennestrom, I. I. Ivanova, J. Phys. Chem. Lett. 2016, 7, 1249.

78 F. Fan, Z. Feng, C. Li, Chem. Soc. Rev. 2010, 39, 4794;

79 J. W. Niemantsverdriet, Spectroscopy in Catalysis, Wiley-VCH Third ed. (2007)

80 S. Bordiga, E. Groppo, G. Agostini, J. A. van Bokhoven, C. Lamberti, Chem. Rev. 2013, 113, 1736.

81 G. M. Lari, P. Y. Dapsens, D. Scholz, S. Mitchell, C. Mondelli, J. Perez-Ramirez, Green Chem. 2015, 18, 1249.

82 J. D. Lewis, S. Van der Vyver, A. J. Crisci, W. R. Gunther, V. K. Michaelis, R. G. Griffin, Y. Román-Leshkov, ChemSusChem, 2014, 7, 2255,

83 M. Chen, D. Kumar, C.-W. Yi, D. W. Goodman, Science, 2005 310, 291.

84 R. C. Tiruvalam, J. C. Pritchard, N. Dimitratos, J. A. LopezSanchez, J. K. Edwards, A. F. Carley, G. J. Hutchings, C. J. Kiely, Faraday Discuss. 2011, 152, 63.

85 E. K. Gibson, A. M. Beale, C. R. A. Catlow, A. Chutia, D. Gianolio, A. Gould, A. Kroner, K. M. H. Mohammed, M. Perdjon, S. M. Rogers, P. P. Wells, Chem. Mater. 2015, 27 , 3714.

86 J. W. Harris, M. J. Cordon, J. R. Di lorio, J. C. Vega-Vila, F. H. Ribeiro, R. Gounder, J. Catal. 2016, 335, 141.

87 M. Morad, M. Sankar, E. Cao, E. Nowicka, T. E. Davies, P. J. Miedziak, D. J. Morgan, D. W. Knight, D. Bothell, A. Gavriilidis, G. J. Hutchings, Cat. Sci. Tech. 2014, 4, 3120.

88 D. M. Alonso, S. G. Wettstein, J. A. Dumesic, Chem. Soc. Rev. 2012, 41, 8075 .

89 A. Cao, R. Lu, G. Veser, Phys. Chem. Chem. Phys. 2010, 12, 13499.

90 P. Mania, R. Verel, F. Jenny, C. Hammond, I. Hermans, Chem Eur. J. 2013, 19, 9849

91 D. Padovan, A. Al Nayili, C. Hammond, submitted manuscript 2017.

92 B. J. O'Neill, J. T. Miller, P. J. Dietrich, X. Gu, F. JG. Sollberger, F. H. Ribeiro, J. A. Dumesic, ChemCatChem 2014, 7, 3247.

93 F. Heroguel, B. Rozmyslowicz, J. S. Luterbacher, Chimia, 2015, 69, 582.

94 C. Aellig, D. Scholz, S. Conrad, I. Hermans, Green Chem. 2013, 15, 1975.

95 W. N. P. van der Graaff, G. Li, B. Mezari, E. A. Pidko, E. J. M. Hensen, ChemCatChem, 2015, 7, 1152.

96 S. Van de Vyver, C. Odermatt, K. Romero, T. Prasomsri, Y. Roman-Leshkov, ACS Catal. 2015, 5, 972.

97 C. Aellig, D. Scholz, P. Y. Dapsens, C. Mondelli, J. PerezRamirez, Catal. Sci. Tech. 2015, 5, 142.

98 R. Gounder, M. E. Davis, AIChE J. 2013, 59, 3349.

99 R. M. Ravanelle, F. Schüßler, A. D'Amico, N. Danilina, J. A. van Bokhoven, J. A. Lercher, C. W. Jones, C. Sievers, J. Phys. Chem. C. 2010, 114, 19582. 
100 H. N. Pham, A. E. Anderson, R. J. Johnson, J. Cardenas, A. Okerlund, N. A. Da Silva, J. Schmidt-Rohr, J. A. Dumesic, Angew. Chem. Int. Ed. 2014, 53, 12718

101 M. Nemanashi, R. Meijboom, Catal. Commun. 2016, 83 53.

102 I. I. Ivanova, Y. G. Kolyagin, ChemSocRev. 2010, 39, 5018.

103 A. Chakrabarti, M. E. Ford, D. Gregory, R. Hu, C. J. Keturakis, S. Lwin, Y. Tang, Z. Yang, M. Zhu, M. A. Banares, I. Wachs, Catal. Today 2017, 283, 27.

104 K. Morgan, J. Touitou, J.-S. Choi, C. Coney, C. Hardacre, J. A. Pihl, C. E. Stere, M.-Y. Kim, C. Stewart, A. Goguet, W. P. Partridge, ACS Catal. 2016, 6, 1356.

105 J.-D. Grunwaldt, S. Hannemann, C. G. Schroer, A. Baiker, J. Phys. Chem. B. 2006, 110, 8674.

106 I. L. C. Buurmans, B. M. Weckhuysen, Nat. Chem. 2012, 4, 873.

107 S. W. T. Price, D. J. Martin, A. D. Parsons, W. A. Stawinski, A. Vamvakeros, S. J. Keylock, A. M. Beale, J. F. W. Mosselmans, Sci. Adv. 2017, 3, 1. 